\title{
Distributed extended Kalman filtering for state-saturated nonlinear systems subject to randomly occurring cyberattacks with uncertain probabilities
}

\author{
Jiaxing $\mathrm{Li}^{1}$, Jun $\mathrm{Hu}^{1,3^{*}}$ (D), Dongyan Chen ${ }^{1,2}$ and Zhihui $\mathrm{Wu}^{1,3}$
}

"Correspondence:

hujun2013@gmail.com

'Department of Mathematics,

Harbin University of Science and

Technology, Harbin 150080, China

${ }^{3}$ School of Engineering, University

of South Wales, Pontypridd CF37

$1 \mathrm{DL}, \mathrm{UK}$

Full list of author information is

available at the end of the article

\begin{abstract}
In this paper, the extended Kalman filtering scheme in a distributed manner is presented for state-saturated nonlinear systems (SSNSs), where the randomly occurring cyberattacks (ROCAs) with uncertain occurring probabilities (UOPs) are taken into account. In particular, a novel cyberattack model is constructed by the consideration of false data-injection attacks (FDIAs) and denial-of-service attacks (DoSAs) simultaneously. The ROCAs are described by a series of Bernoulli distributed stochastic variables, where the so-called UOPs are considered and described by the nominal mathematical expectations and error bounds. The major effort is to develop a novel DEKF strategy for SSNSs with consideration of state delay and ROCAs with UOPs. In what follows, an upper bound with respect to the filtering error covariance is derived and minimized by selecting the suitable filter parameter. Besides, the concrete expression of the filter parameter is formed by solving matrix difference equations (MDEs). Meanwhile, a sufficient condition under certain constraints is proposed to testify the boundedness regarding the given upper bound. Finally, we use the experiments and corresponding comparisons to verify the feasibility of the designed extended Kalman filtering approach in a distributed way.
\end{abstract}

Keywords: Distributed extended Kalman filtering; State-saturated systems; Time delay; Uncertain occurring probabilities; Randomly occurring cyberattacks

\section{Introduction}

During the past few decades, the dynamical networks have been extensively applied to the modeling in a wealth of areas such as air cooperative monitoring, seismic sensing, target tracking and so forth, see, e.g., [1-6] for more details. Accordingly, a wireless sensor network (WSN) comprises a majority of intelligent nodes, which are capable of sensing, monitoring, collecting data information, and communicating with their adjacent nodes at the same time. In order to reduce production costs, each intelligent node normally has small size and limited power in practical engineering systems. Thus, the traditional filtering method, which requires that every node transmits the information to the fusion center or other sensor nodes, has certain application limitations $[7,8]$. To handle this issue, the

(c) The Author(s) 2020. This article is licensed under a Creative Commons Attribution 4.0 International License, which permits use sharing, adaptation, distribution and reproduction in any medium or format, as long as you give appropriate credit to the original author(s) and the source, provide a link to the Creative Commons licence, and indicate if changes were made. The images or other third party material in this article are included in the article's Creative Commons licence, unless indicated otherwise in a credit line to the material. If material is not included in the article's Creative Commons licence and your intended use is not permitted by statutory regulation or exceeds the permitted use, you will need to obtain permission directly from the copyright holder. To view a copy of this licence, visit http://creativecommons.org/licenses/by/4.0/. 
distributed filtering (DF) whose core idea is to estimate the target's state on account of the local information performs better to some extent. To name a few works in this area, the $H_{\infty}$ filtering problem in a distributed setting has been tackled in [9] for Markovian jump systems by solving some matrix inequalities. In addition, the DF schemes with fading measurements and redundant channels have been presented in [10] and [11], respectively. And in [12], the DF issue has been addressed for nonlinear systems with switching topology and multiplicative link noises, where the filter parameters have been obtained by means of handling convex optimization problems.

It should be noted that the state saturation occurs frequently in the practical engineering, which can reflect the limitation of systems' state or the protection of the machinery equipment. Generally speaking, the state variables of the physical target are constrained in a bounded set $[13,14]$. If the state saturation is not aptly addressed, the desired performance of dynamic systems cannot be always ensured. So far, many efforts have been devoted to the studies of synthesis and analysis for state saturated systems $[15,16]$. For instance, the state-saturated filtering issue has been solved in [17] via certain mathematical computations. In [18], a set-membership filtering algorithm has been studied for statesaturated systems in light of dealing with some linear matrix inequalities. The DF strategy has been proposed in [19] for state-saturated systems under fading measurements and quantization in terms of the feasibility of certain matrix difference equations (MDEs). Moreover, the nonlinearities are ubiquitous in actual world, which should be tackled in a proper way [20-24]. For instance, a new filtering algorithm by considering the event-based communication criterion has been proposed in [25] for nonlinear systems. Nevertheless, it should be noted that few approaches are available to tackle the DF problem for timevarying saturated nonlinear systems, which is one motivation of this paper.

Accompanied with the broad utilizations of open but unprotected shared networks, the sensor networks are extremely vulnerable to attacks, see, e.g., [26, 27]. In fact, the fundamental goal of these attacks is to break data information through the shared communication network so as to drive the physical plant to become oscillating or even instable. In [28], the recursive filtering method has been proposed for state-saturated systems under deception attacks, where the attacks have been characterized by a series of Bernoulli stochastic variables with a known occurrence probability. It is worthwhile to notice that the behaviors of attacks might be irregularly intercepted by the protection equipment, namely the attacks generally occur in a random manner. In [29], the extended Kalman filtering issue has been addressed for stochastic systems under randomly occurring cyberattacks (ROCAs). Unfortunately, it should be pointed out that the occurrence probability of the attacks is commonly supposed to be deterministic among the existing results, where such a situation cannot be guaranteed always and restricts its applications. Recently, the recursive filtering method under the variance-constraint criterion has been developed in [30] to attenuate the impacts caused by multiple missing measurements with uncertain missing probabilities. However, there are few studies about the cyberattacks with uncertain occurring probabilities (UOPs) and the state saturation in the existing literature, which deserves further investigations in order to develop the corresponding filtering algorithms with potential robustness. Moreover, time delay unavoidably exists in practical systems, see, e.g., [31-33]. Accordingly, the performance of the system might be destroyed if the time delay cannot be effectively handled [34,35]. In [36], the variance-constrained filtering method has been established for stochastic genetic regulatory networks subject to state 
delay. Nevertheless, it should be noticed that few methods can be found that handle the delayed time-varying stochastic systems with state saturation, not to mention the presence of ROCAs with UOPs. Hence, the goal of this paper is to fill such a gap by designing a novel distributed extended Kalman filtering (DEKF) strategy for the addressed problem. Inspired by the aforementioned discussions, we attempt to provide a DEKF algorithm for state-saturated nonlinear systems (SSNSs) subject to state delay and ROCAs with UOPs. The phenomena of cyberattacks are adequately considered and characterized by a sequence of nominal means, error upper bounds, as well as the bound of the attack signal. The key motivations/challenges can be mentioned from three aspects: (i) How to comprehensively take the state saturation, time-delay and ROCAs with UOPs into account when conducting the design problem of DEKF? (ii) How to design the proper filter gain with an easy-to-implement form to realize the expected DEKF performance requirement? (iii) How to evaluate the estimation method performance and ensure the boundedness requirement regarding the upper bound matrix of the filtering error covariance. In order to better answer the above questions, an effective optimal DEKF method is established, where the accurate expression of the filter gain is presented by solving two MDEs. The main contributions can be summarized as follows: (1) the constructed dynamic system model is fairly comprehensive and contains nonlinearity disturbance, state saturation, time-delay, and ROCAs with UOPs, thus better reflecting the practical engineering situations; (2) a matrix simplification technology is exploited to cope with the design difficulty of filter gain caused by the sparseness of the fixed topology; (3) a sufficient condition is devised to guarantee the boundedness of the proposed upper bound matrix; and (4) the newly proposed DEKF scheme can be applied for online implementations. Finally, the usefulness of the main results is certified by a few simulations.

Now, we outline the rest of the paper. The DEKF problem is formulated in Sect. 2 for state-saturated systems under a given network topology. In Sect. 3, the upper bound for the state estimation error covariance is obtained firstly and then the proper filter parameter is chosen to minimize such an upper bound. Moreover, the boundedness is analyzed and the proof is provided under an assumption. Two numerical simulations are utilized in Sect. 4 to demonstrate the usefulness of our main theoretical results. Finally, we summarize the conclusions in Sect. 5.

Notations The symbols utilized throughout the paper are standard. The $n$-dimensional Euclidean space is denoted by $\mathbb{R}^{n}$. The symbol $C \geq D$, where $C$ and $D$ are both symmetric matrices, implies that $C-D$ is positive semi-definite. For a matrix $W,\|W\|, \rho(W), W^{T}$, $\sigma_{\max }(W)$ and $\operatorname{tr}\{W\}$ stand for the spectral norm of matrix $W$, the spectral radius of matrix $W$, the transpose of matrix $W$, the largest singular value of matrix $W$, and the trace of matrix $W$, respectively; $\operatorname{col}\left\{W_{1}, W_{2}, \ldots, W_{N}\right\}$ represents $\left[\begin{array}{llll}W_{1}^{T} & W_{2}^{T} & \ldots & W_{N}^{T}\end{array}\right]^{T}$; o denotes the Hadamard product operator, with $[P \circ Q]_{i j}=P_{i j} Q_{i j} ; \operatorname{diag}\{\cdot\}$ is a block-diagonal matrix; $I$ represents an identity matrix with proper dimension. Furthermore, matrices are assumed to have suitable dimensions for the mathematical operations if they are not explicitly pointed out.

\section{Problem formulation}

The DEKF problem with attacks is drawn in Fig. 1. From the diagram, it is easy to see that the outputs are measured by some sensor nodes. Meanwhile, the information can be 


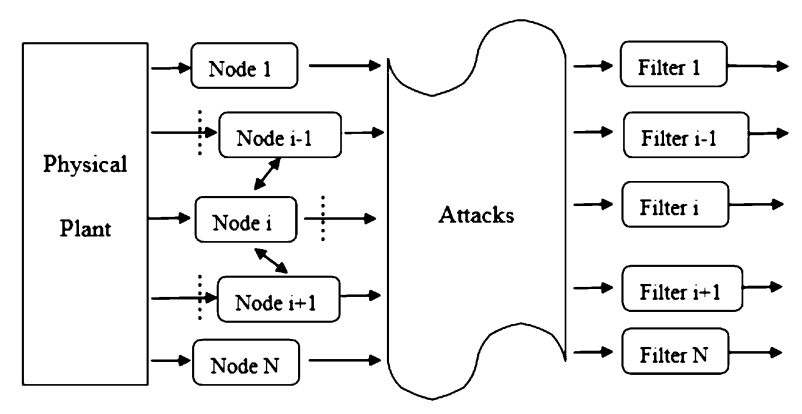

Figure 1 The DEKF problem under attacks

collected from the individual and the neighboring nodes according to a certain topological structure. Additionally, the cyberattacks randomly occur in the shared communication networks between the sensors and the remote filters.

The topological structure of the WSN is modeled by a graph $\mathcal{G}=(\mathcal{U}, \mathcal{E}, \mathcal{A})$, where the set of nodes, the set of edges, as well as the weighted adjacency matrix are denoted by $\mathcal{U}=\{1,2, \ldots, N\}, \mathcal{E} \subseteq \mathcal{U} \times \mathcal{U}$, and $\mathcal{A}=\left[\alpha_{i j}\right]_{N \times N}$ with $\alpha_{i j}>0$, respectively. An edge of $\mathcal{G}$ is given by $(i, j)$. Also $\alpha_{i j}>0 \Longleftrightarrow(i, j) \in \mathcal{E}$ which shows that there is an edge from $j$ to $i$. Besides, $\mathcal{N}_{i}=\left\{j \in \mathcal{U}: \alpha_{i j}>0\right\}$ represents the set of adjacent nodes for node $i$, including itself.

In this paper, the considered SSNSs are described by the following dynamical equations:

$$
\begin{aligned}
& x_{k+1}=\mu\left(A_{k} x_{k}+f\left(x_{k-d}\right)\right)+B_{k} \omega_{k}, \\
& \mathbf{y}_{i, k}=h_{i}\left(x_{k}\right)+v_{i, k},
\end{aligned}
$$

where $x_{k} \in \mathbb{R}^{n}$ depicts the state vector that cannot be directly measured, the initial value of $x_{k}$ has mean $\bar{x}_{0}$ and covariance $P_{0}$, and $\mathbf{y}_{i, k} \in \mathbb{R}^{m_{y}}(i=1,2, \ldots, N)$ is the output signal collected by sensors. The random parameters $\omega_{k} \in \mathbb{R}^{n_{w}}$ and $v_{i, k} \in \mathbb{R}^{m_{y}}$ are the process and measurement noises with zero mean and known covariances, $R_{k}>0$ and $Q_{i, k}>0$, respectively. The known positive integer $d$ depicts the time delay. The system matrices $A_{k}$ and $B_{k}$ are known with proper dimensions. The nonlinearities $f(\cdot)$ and $h_{i}(\cdot)$ are both supposed to be continuously differentiable.

The nonlinearity $h_{i}\left(x_{k}\right): \mathbb{R}^{n} \mapsto \mathbb{R}^{m_{y}}$ satisfies the following condition:

$$
\left\|h_{i}\left(x_{k}\right)\right\| \leq \delta_{1}\left\|x_{k}\right\|+\delta_{2}
$$

where $\delta_{1}$ and $\delta_{2}$ are nonnegative scalars.

The saturation function $\mu(\cdot): \mathbb{R}^{n} \mapsto \mathbb{R}^{n}$ can be defined as

$$
\mu(\varsigma)=\left[\begin{array}{llll}
\mu_{1}\left(\varsigma_{1}\right) & \mu_{2}\left(\varsigma_{2}\right) & \cdots & \mu_{n}\left(\varsigma_{n}\right)
\end{array}\right]^{T},
$$

where

$$
\mu_{i}\left(\varsigma_{i}\right)=\operatorname{sign}\left(\varsigma_{i}\right) \min \left\{\varsigma_{i, \max },\left|\varsigma_{i}\right|\right\},
$$


$\varsigma_{i, \max }$ stands for the $i$ th element of the saturation level $\varsigma_{\max }, \varsigma_{i}$ denotes the real value, and $\mu_{i}\left(\varsigma_{i}\right)$ represents the value under the saturation constraint.

The real measured output of the $i$ th node, which might be subject to ROCAs, is modeled by

$$
y_{i, k}=\gamma_{i, k} \mathbf{y}_{i, k}+\left(1-\gamma_{i, k}\right) \lambda_{i, k} \xi_{i, k}
$$

where $y_{i, k} \in \mathbb{R}^{m_{y}}$ stands for the output signal with potential attacks; $\xi_{i, k} \in \mathbb{R}^{m_{y}}$ is the attack signal transmitted by malicious attackers satisfying $\xi_{i, k}^{T} \xi_{i, k} \leq \bar{\xi}_{k}$. The mutually independent Bernoulli distributed variables $\gamma_{i, k}$ and $\lambda_{i, k}$ are utilized to describe the switch behavior of different cyberattacks, where the statistical features are given as:

$$
\begin{array}{ll}
\operatorname{Prob}\left\{\lambda_{i, k}=1\right\}=\bar{\lambda}_{i, k}+\Delta \lambda_{i, k}, & \operatorname{Prob}\left\{\lambda_{i, k}=0\right\}=1-\left(\bar{\lambda}_{i, k}+\Delta \lambda_{i, k}\right), \\
\operatorname{Prob}\left\{\gamma_{i, k}=1\right\}=\bar{\gamma}_{i, k}+\Delta \gamma_{i, k}, & \operatorname{Prob}\left\{\gamma_{i, k}=0\right\}=1-\left(\bar{\gamma}_{i, k}+\Delta \gamma_{i, k}\right),
\end{array}
$$

where $\bar{\gamma}_{i, k}+\Delta \gamma_{i, k}$ and $\bar{\lambda}_{i, k}+\Delta \lambda_{i, k}$ both belong to $[0,1], \bar{\gamma}_{i, k}$ and $\bar{\lambda}_{i, k}$ are known scalars, $\Delta \gamma_{i, k}$ and $\Delta \lambda_{i, k}$ are introduced to describe the UOPs, $\left|\Delta \gamma_{i, k}\right| \leq \rho_{i, k}$ and $\left|\Delta \lambda_{i, k}\right| \leq \bar{\rho}_{i, k}$ with $\rho_{i, k}$ and $\bar{\rho}_{i, k}$ being known scalars. Furthermore, we assume that $\gamma_{i, k}, \lambda_{i, k}, \omega_{k}$, and $v_{i, k}$ are mutually uncorrelated for any $i$ and $k$.

Remark 1 In accordance with (6), the different values of $\lambda_{i, k}$ and $\gamma_{i, k}$ can describe different cases. To be more specific, it is easy to see that the measurement output model in (6) mainly includes the following three cases: (1) if $\gamma_{i, k}=1$, the measurement signals can be transmitted successfully, i.e., the behaviors of attacks cannot occur; (2) when $\gamma_{i, k}=0$ and $\lambda_{i, k}=1$, the systems encounter the false data-injection attacks (FDIAs); and (3) when $\gamma_{i, k}=$ 0 and $\lambda_{i, k}=0$, the systems suffer from the denial-of-service attacks (DoSAs).

Remark 2 On the one hand, it is worth noticing that the UOPs are characterized by (7) and (8), where $\Delta \gamma_{i, k}$ and $\Delta \lambda_{i, k}$ denote the UOPs subject to the certain probability error bounds with a hope to reflect the practical engineering environments. On the other hand, the fuzzy uncertainty method can be considered. In fact, the choice of membership functions is crucial for the realization of purpose, which is commonly dependent on the experience for different systems. Compared with the modeling of fuzzy uncertainty, we adopt another method to model the UOPs described by the nominal means and error upper bounds. Moreover, the new modeling way contains the existing ROCAs as a special one when setting $\Delta \lambda_{i, k}=0$ and $\Delta \gamma_{i, k}=0$.

The following distributed filter is designed for addressed SSNSs:

$$
\begin{aligned}
& \hat{x}_{i, k+1 \mid k}=\mu\left(A_{k} \hat{x}_{i, k \mid k}+f\left(\hat{x}_{i, k-d \mid k-d}\right)\right), \\
& \hat{x}_{i, k+1 \mid k+1}=\hat{x}_{i, k+1 \mid k}+\sum_{j \in \mathcal{N}_{i}} \alpha_{i j} G_{i j, k+1}\left(y_{j, k+1}-\bar{\gamma}_{j, k+1} h_{j}\left(\hat{x}_{j, k+1 \mid k}\right)\right),
\end{aligned}
$$

where $\hat{x}_{i, k+1 \mid k}$ is the one-step prediction, $\hat{x}_{i, k+1 \mid k+1}$ is the filter. In the sequel, the matrix $G_{i j, k+1}$ is the desirable time-varying filter parameter. 
Now, we devote to state the major objectives of this paper. Firstly, a new DEKF method is proposed for SSNSs under ROCAs with UOPs. Secondly, the boundedness is analyzed for the newly designed DEKF scheme.

Before proceeding, we provide the following lemmas, which are helpful for the further theoretical derivations.

Lemma 1 ([11]) For $\forall u, v \in \mathbb{R}$, there is a scalar $\epsilon_{i} \in[0,1]$ satisfying

$$
\mu_{i}(u)-\mu_{i}(v)=\epsilon_{i}(u-v), \quad i=1,2, \ldots, N,
$$

where $\mu_{i}(\cdot)$ is defined in (4)-(5).

Lemma 2 ([19]) Let $N=\operatorname{diag}\left\{n_{1}, n_{2}, \ldots, n_{p}\right\}$ be a random diagonal matrix and $M=$ $\left[m_{i j}\right]_{p \times p}$ be a real-valued matrix. Then, it can shown that the following relationship holds:

$$
\mathbb{E}\left\{N M N^{T}\right\}=\left[\begin{array}{cccc}
\mathbb{E}\left\{n_{1}^{2}\right\} & \mathbb{E}\left\{n_{1} n_{2}\right\} & \cdots & \mathbb{E}\left\{n_{1} n_{p}\right\} \\
\mathbb{E}\left\{n_{2} n_{1}\right\} & \mathbb{E}\left\{n_{2}^{2}\right\} & \cdots & \mathbb{E}\left\{n_{2} n_{p}\right\} \\
\vdots & \vdots & \ddots & \vdots \\
\mathbb{E}\left\{n_{p} n_{1}\right\} & \mathbb{E}\left\{n_{p} n_{2}\right\} & \cdots & \mathbb{E}\left\{n_{p}^{2}\right\}
\end{array}\right] \circ M
$$

Lemma 3 ([29]) Consider matrices $\mathcal{C}, \mathcal{M}, \wp$, and an unknown matrix $\mathcal{F}$ satisfying $\mathcal{F F}^{T} \leq I$. If there are $\aleph>0$ and $\zeta>0$ such that $\zeta^{-1} I-\wp \aleph \wp \wp^{T}>0$, then we have

$$
(\mathcal{C}+\mathcal{M F} \mathcal{F} \wp)(\mathcal{C}+\mathcal{M} \mathcal{F} \wp)^{T} \leq \mathcal{C}\left(\aleph^{-1}-\zeta \wp^{T} \wp\right)^{-1} \mathcal{C}^{T}+\zeta^{-1} \mathcal{M} \mathcal{M}^{T}
$$

\section{DEKF algorithm design and error boundedness discussion}

In this section, a DEKF algorithm will be given for SSNSs subject to state delay and ROCAs with UOPs. Firstly, we analyze the nonlinearities $f\left(x_{k-d}\right)$ and $h_{j}\left(x_{k+1}\right)$ by employing the Taylor series expansion, where the high-order terms induced by the linearization process are approximately estimated. Secondly, a major effort is made on looking for a minimal upper bound matrix with respect to the state estimation error covariance, where the matrix simplification technique (MST) is adopted to obtain the proper filter parameter with an easy-to-implement manner. Finally, the performance evaluation issue is addressed, where a new sufficient criterion under certain constraint conditions is given in order to characterize the boundedness of the estimation error covariance.

\subsection{DEKF algorithm design}

To begin, the prediction error and filtering error are expressed as

$$
e_{i, k+1 \mid k}=x_{k+1}-\hat{x}_{i, k+1 \mid k}, \quad e_{i, k+1 \mid k+1}=x_{k+1}-\hat{x}_{i, k+1 \mid k+1} .
$$

Then, in light of the Taylor series expansion for $f\left(x_{k-d}\right)$ around $\hat{x}_{i, k-d \mid k-d}$, one has

$$
f\left(x_{k-d}\right)=f\left(\hat{x}_{i, k-d \mid k-d}\right)+E_{i, k-d} e_{i, k-d \mid k-d}+o\left(\left|e_{i, k-d \mid k-d}\right|\right),
$$


where

$$
E_{i, k-d}=\left.\frac{\partial f\left(x_{k-d}\right)}{\partial x_{k-d}}\right|_{x_{k-d}=\hat{x}_{i, k-d \mid k-d}} .
$$

Moreover, the high-order term $o\left(\left|e_{i, k-d \mid k-d}\right|\right)$ by using the Taylor series expansion is approximately expressed by

$$
o\left(\left|e_{i, k-d \mid k-d}\right|\right)=B_{i, k-d} \Theta_{i, k-d} e_{i, k-d \mid k-d}
$$

where $B_{i, k-d}$ is supposed to be a scaling matrix with regard to the specific issue and $\Theta_{i, k-d}$ is an unknown matrix that symbols the linearization error with $\Theta_{i, k-d} \Theta_{i, k-d}^{T} \leq I$.

Along the same lines, the following equation can be obtained:

$$
h_{j}\left(x_{k+1}\right)=h_{j}\left(\hat{x}_{j, k+1 \mid k}\right)+F_{j, k+1} e_{j, k+1 \mid k}+o\left(\left|e_{j, k+1 \mid k}\right|\right) \text {, }
$$

where $F_{j, k+1}=\left.\frac{\partial h_{j}\left(x_{k+1}\right)}{\partial x_{k+1}}\right|_{x_{k+1}=\hat{x}_{j, k+1 \mid k}}, o\left(\left|e_{j, k+1 \mid k}\right|\right)=C_{j, k+1} \Omega_{j, k+1} e_{j, k+1 \mid k}$, and $\Omega_{j, k+1} \Omega_{j, k+1}^{T} \leq I$.

From Lemma 1, (1), (9), and (11)-(12), we have

$$
e_{i, k+1 \mid k}=\Pi_{i, k} A_{k} e_{i, k \mid k}+\Pi_{i, k}\left(E_{i, k-d}+B_{i, k-d} \Theta_{i, k-d}\right) e_{i, k-d \mid k-d}+B_{k} \omega_{k}
$$

where $\Pi_{i, k}=\operatorname{diag}\left\{\epsilon_{i, k}^{(1)}, \epsilon_{i, k}^{(2)}, \ldots, \epsilon_{i, k}^{(n)}\right\}, \epsilon_{i, k}^{(\varsigma)} \in[0,1](\varsigma=1,2, \ldots, n)$.

Together with (1), (10), and (13), we have

$$
\begin{aligned}
e_{i, k+1 \mid k+1}= & e_{i, k+1 \mid k}-\sum_{j \in \mathcal{N}_{i}} \alpha_{i j} G_{i j, k+1}\left\{\left(\gamma_{j, k+1}-\bar{\gamma}_{j, k+1}-\Delta \gamma_{j, k+1}\right) h_{j}\left(x_{k+1}\right)\right. \\
& +\bar{\gamma}_{j, k+1}\left(F_{j, k+1}+C_{j, k+1} \Omega_{j, k+1}\right) e_{j, k+1 \mid k}+\left[\left(1-\gamma_{j, k+1}\right) \lambda_{j, k+1}\right. \\
& \left.-\left(1-\bar{\gamma}_{j, k+1}-\Delta \gamma_{j, k+1}\right)\left(\bar{\lambda}_{j, k+1}+\Delta \lambda_{j, k+1}\right)\right] \xi_{j, k+1} \\
& +\left(1-\bar{\gamma}_{j, k+1}-\Delta \gamma_{j, k+1}\right)\left(\bar{\lambda}_{j, k+1}+\Delta \lambda_{j, k+1}\right) \xi_{j, k+1} \\
& \left.+\gamma_{j, k+1} v_{j, k+1}+\Delta \gamma_{j, k+1} h_{j}\left(x_{k+1}\right)\right\} .
\end{aligned}
$$

To facilitate subsequent derivations, we define

$$
\begin{aligned}
& e_{k \mid k}=\operatorname{col}_{N}\left\{e_{i, k \mid k}\right\}, \quad e_{k-d \mid k-d}=\operatorname{col}_{N}\left\{e_{i, k-d \mid k-d}\right\}, \quad \bar{\Xi}=\max \left\{\bar{\xi}_{k}\right\}, \\
& e_{k+1 \mid k}=\operatorname{col}_{N}\left\{e_{i, k+1 \mid k}\right\}, \quad \Lambda_{k}=\operatorname{diag}_{N}\left\{\lambda_{i, k} I\right\}, \quad \bar{A}_{k}=\operatorname{diag}_{N}\left\{A_{k}\right\}, \\
& \bar{B}_{k}=\operatorname{col}_{N}\left\{B_{k}\right\}, \quad \bar{x}_{k}=\operatorname{col}_{N}\left\{x_{k}\right\}, \quad T_{i}=\operatorname{diag}\left\{\alpha_{i 1} I, \ldots, \alpha_{i N} I\right\}, \\
& Q_{k}=\operatorname{diag}_{N}\left\{Q_{i, k}\right\}, \quad E_{k-d}=\operatorname{diag}_{N}\left\{E_{i, k-d}\right\}, \quad \Theta_{k-d}=\operatorname{diag}_{N}\left\{\Theta_{i, k-d}\right\}, \\
& B_{k-d}=\operatorname{diag}_{N}\left\{B_{i, k-d}\right\}, \quad F_{k+1}=\operatorname{diag}_{N}\left\{F_{i, k+1}\right\}, \quad v_{k}=\operatorname{col}_{N}\left\{v_{i, k}\right\}, \\
& C_{k+1}=\operatorname{diag}_{N}\left\{C_{i, k+1}\right\}, \quad \Omega_{k+1}=\operatorname{diag}_{N}\left\{\Omega_{i, k+1}\right\}, \quad \Pi_{k}=\operatorname{diag}_{N}\left\{\Pi_{i, k}\right\}, \\
& \Xi_{k}=\operatorname{col}_{N}\left\{\xi_{i, k}\right\}, \quad \Gamma_{k}=\operatorname{diag}_{N}\left\{\gamma_{i, k} I\right\}, \quad \bar{h}\left(x_{k+1}\right)=\operatorname{col}_{N}\left\{h_{i}\left(x_{k+1}\right)\right\}, \\
& H_{i}=\operatorname{diag}\{\underbrace{0, \ldots, 0}_{i-1}, I, \underbrace{0, \ldots, 0}_{N-i}\}, \quad G_{k}=\left[G_{i j, k}\right]_{N \times N} .
\end{aligned}
$$


Then, the one-step prediction error in (14) and filtering error in (15) in the compact form can be described by:

$$
\begin{aligned}
e_{k+1 \mid k}=\Pi_{k} & \bar{A}_{k} e_{k \mid k}+\Pi_{k}\left(E_{k-d}+B_{k-d} \Theta_{k-d}\right) e_{k-d \mid k-d}+\bar{B}_{k} \omega_{k}, \\
e_{k+1 \mid k+1}= & {\left[I-\sum_{i=1}^{N} H_{i} G_{k+1} T_{i} \bar{\Gamma}_{k+1}\left(F_{k+1}+C_{k+1} \Omega_{k+1}\right)\right] e_{k+1 \mid k} } \\
& -\sum_{i=1}^{N} H_{i} G_{k+1} T_{i} \tilde{\Gamma}_{k+1} \bar{h}\left(x_{k+1}\right)-\sum_{i=1}^{N} H_{i} G_{k+1} T_{i} \check{\Gamma}_{k+1} \bar{h}\left(x_{k+1}\right) \\
& -\sum_{i=1}^{N} H_{i} G_{k+1} T_{i}\left[\left(I-\Gamma_{k+1}\right) \Lambda_{k+1}-\left(I-\hat{\Gamma}_{k+1}\right) \hat{\Lambda}_{k+1}\right] \Xi_{k+1} \\
& -\sum_{i=1}^{N} H_{i} G_{k+1} T_{i} \Gamma_{k+1} v_{k+1}-\sum_{i=1}^{N} H_{i} G_{k+1} T_{i}\left(I-\hat{\Gamma}_{k+1}\right) \hat{\Lambda}_{k+1} \Xi_{k+1},
\end{aligned}
$$

where

$$
\begin{aligned}
\tilde{\Gamma}_{k+1}= & \Gamma_{k+1}-\hat{\Gamma}_{k+1}, \\
\hat{\Gamma}_{k+1}= & \operatorname{diag}\left\{\left(\bar{\gamma}_{1, k+1}+\Delta \gamma_{1, k+1}\right) I,\left(\bar{\gamma}_{2, k+1}+\Delta \gamma_{2, k+1}\right) I,\right. \\
& \left.\ldots,\left(\bar{\gamma}_{N, k+1}+\Delta \gamma_{N, k+1}\right) I\right\}, \\
\check{\Gamma}_{k+1}= & \operatorname{diag}\left\{\Delta \gamma_{1, k+1} I, \Delta \gamma_{2, k+1} I, \ldots, \Delta \gamma_{N, k+1} I\right\}, \\
\bar{\Gamma}_{k+1}= & \operatorname{diag}\left\{\bar{\gamma}_{1, k+1} I, \bar{\gamma}_{2, k+1} I, \ldots, \bar{\gamma}_{N, k+1} I\right\}, \\
\tilde{\Lambda}_{k+1}= & \Lambda_{k+1}-\hat{\Lambda}_{k+1}, \\
\hat{\Lambda}_{k+1}= & \operatorname{diag}\left\{\left(\bar{\lambda}_{1, k+1}+\Delta \lambda_{1, k+1}\right) I,\left(\bar{\lambda}_{2, k+1}+\Delta \lambda_{2, k+1}\right) I,\right. \\
& \left.\ldots,\left(\bar{\lambda}_{N, k+1}+\Delta \lambda_{N, k+1}\right) I\right\}, \\
\check{\Lambda}_{k+1}= & \operatorname{diag}\left\{\Delta \lambda_{1, k+1} I, \Delta \lambda_{2, k+1} I, \ldots, \Delta \lambda_{N, k+1} I\right\}, \\
\bar{\Lambda}_{k+1}= & \operatorname{diag}\left\{\bar{\lambda}_{1, k+1} I, \bar{\lambda}_{2, k+1} I, \ldots, \bar{\lambda}_{N, k+1} I\right\} .
\end{aligned}
$$

Subsequently, we will give the concrete forms of $P_{k+1 \mid k}=\mathbb{E}\left\{e_{k+1 \mid k} e_{k+1 \mid k}^{T}\right\}$ and $P_{k+1 \mid k+1}=$ $\mathbb{E}\left\{e_{k+1 \mid k+1} e_{k+1 \mid k+1}^{T}\right\}$, where the detailed calculation derivations are omitted for conciseness.

Lemma 4 The prediction error covariance matrix $P_{k+1 \mid k}$ can be shown to be

$$
P_{k+1 \mid k}=\mathbb{E}\left\{\Pi_{k} \bar{A}_{k} e_{k \mid k} e_{k \mid k}^{T} \bar{A}_{k}^{T} \Pi_{k}^{T}\right\}+\mathcal{A}_{k}+\bar{B}_{k} R_{k} \bar{B}_{k}^{T}+\mathcal{B}_{k}+\mathcal{B}_{k}^{T}
$$

where

$$
\begin{aligned}
& \mathcal{A}_{k}=\mathbb{E}\left\{\Pi_{k}\left(E_{k-d}+B_{k-d} \Theta_{k-d}\right) e_{k-d \mid k-d} e_{k-d \mid k-d}^{T}\left(E_{k-d}+B_{k-d} \Theta_{k-d}\right)^{T} \Pi_{k}^{T}\right\}, \\
& \mathcal{B}_{k}=\mathbb{E}\left\{\Pi_{k} \bar{A}_{k} e_{k \mid k} e_{k-d \mid k-d}^{T}\left(E_{k-d}+B_{k-d} \Theta_{k-d}\right)^{T} \Pi_{k}^{T}\right\} .
\end{aligned}
$$


Lemma 5 The filtering error covariance matrix $P_{k+1 \mid k+1}$ obeys

$P_{k+1 \mid k+1}$

$$
\begin{aligned}
= & \mathbb{E}\left\{\left[I-\sum_{i=1}^{N} H_{i} G_{k+1} T_{i} \bar{\Gamma}_{k+1}\left(F_{k+1}+C_{k+1} \Omega_{k+1}\right)\right] e_{k+1 \mid k} e_{k+1 \mid k}^{T}\right. \\
& \left.\times\left[I-\sum_{i=1}^{N} H_{i} G_{k+1} T_{i} \bar{\Gamma}_{k+1}\left(F_{k+1}+C_{k+1} \Omega_{k+1}\right)\right]^{T}\right\} \\
& +\mathbb{E}\left\{\left(\sum_{i=1}^{N} H_{i} G_{k+1} T_{i} \tilde{\Gamma}_{k+1}\right) \bar{h}\left(x_{k+1}\right) \bar{h}^{T}\left(x_{k+1}\right)\left(\sum_{i=1}^{N} H_{i} G_{k+1} T_{i} \tilde{\Gamma}_{k+1}\right)^{T}\right\} \\
& +\mathbb{E}\left\{\left(\sum_{i=1}^{N} H_{i} G_{k+1} T_{i} \check{\Gamma}_{k+1}\right) \bar{h}\left(x_{k+1}\right) \bar{h}^{T}\left(x_{k+1}\right)\left(\sum_{i=1}^{N} H_{i} G_{k+1} T_{i} \check{\Gamma}_{k+1}\right)^{T}\right\} \\
& +\mathbb{E}\left\{\left(\sum_{i=1}^{N} H_{i} G_{k+1} T_{i}\left[\left(I-\Gamma_{k+1}\right) \Lambda_{k+1}-\left(I-\hat{\Gamma}_{k+1}\right) \hat{\Lambda}_{k+1}\right]\right)\right. \\
& \left.\times \Xi_{k+1} \Xi_{k+1}^{T}\left(\sum_{i=1}^{N} H_{i} G_{k+1} T_{i}\left[\left(I-\Gamma_{k+1}\right) \Lambda_{k+1}-\left(I-\hat{\Gamma}_{k+1}\right) \hat{\Lambda}_{k+1}\right]\right)^{T}\right\} \\
& \left.+\mathbb{E}\left\{\left(\sum_{i=1}^{N} H_{i} G_{k+1} T_{i} \Gamma_{k+1}\right) v_{k+1} v_{k+1}^{T}\left(\sum_{i=1}^{N} H_{i} G_{k+1} T_{i} \Gamma_{k+1}\right)\right)^{T}\right\} \\
& +\mathbb{E}\left\{\left[\sum_{i=1}^{N} H_{i} G_{k+1} T_{i}\left(I-\hat{\Gamma}_{k+1}\right) \hat{\Lambda}_{k+1}\right] \Xi_{k+1} \Xi_{k+1}^{T}\right. \\
& \left.\times\left[\sum_{i=1}^{N} H_{i} G_{k+1} T_{i}\left(I-\hat{\Gamma}_{k+1}\right) \hat{\Lambda}_{k+1}\right]^{T}\right\}-\mathcal{C}_{k+1}-\mathcal{C}_{k+1}^{T} \mathcal{D}_{k+1}^{T}+\mathcal{E}_{k+1}+\mathcal{E}_{k+1}^{T},
\end{aligned}
$$

where

$$
\begin{aligned}
\mathcal{C}_{k+1}= & \mathbb{E}\left\{\left[I-\sum_{i=1}^{N} H_{i} G_{k+1} T_{i} \bar{\Gamma}_{k+1}\left(F_{k+1}+C_{k+1} \Omega_{k+1}\right)\right] e_{k+1 \mid k} \bar{h}^{T}\left(x_{k+1}\right)\right. \\
& \left.\times\left(\sum_{i=1}^{N} H_{i} G_{k+1} T_{i} \check{\Gamma}_{k+1}\right)^{T}\right\}, \\
\mathcal{D}_{k+1}= & \mathbb{E}\left\{\left[I-\sum_{i=1}^{N} H_{i} G_{k+1} T_{i} \bar{\Gamma}_{k+1}\left(F_{k+1}+C_{k+1} \Omega_{k+1}\right)\right] e_{k+1 \mid k} \Xi_{k+1}^{T}\right. \\
& \left.\times\left[\sum_{i=1}^{N} H_{i} G_{k+1} T_{i}\left(I-\hat{\Gamma}_{k+1}\right) \hat{\Lambda}_{k+1}\right]^{T}\right\}, \\
\mathcal{E}_{k+1}= & \mathbb{E}\left\{\sum_{i=1}^{N} H_{i} G_{k+1} T_{i} \check{\Gamma}_{k+1} \bar{h}\left(x_{k+1}\right) \Xi_{k+1}^{T}\left[\sum_{i=1}^{N} H_{i} G_{k+1} T_{i}\left(I-\hat{\Gamma}_{k+1}\right) \hat{\Lambda}_{k+1}\right]^{T}\right\} .
\end{aligned}
$$


Note that the uncertain terms exist in (18)-(19), and hence it is impossible to get their precise values. In the sequel, an alternative method is utilized, where a recursive method will be given to obtain the optimized upper bound matrix by designing a suitable filter gain.

Theorem 1 For the given system (1), (2), and (6), let $\varepsilon_{1}>0, \varepsilon_{2}>0$, and $\theta_{\kappa}>0(\kappa=1,2, \ldots, 5)$ be given. If there exist $\Sigma_{k+1 \mid k}>0$ and $\Sigma_{k+1 \mid k+1}>0$ satisfying initial condition $\Sigma_{0 \mid 0}=P_{0 \mid 0}>0$ and meeting the following MDEs:

$$
\Sigma_{k+1 \mid k}=\min \left\{U_{k}, 4 N \hbar\right\} I+\bar{B}_{k} R_{k} \bar{B}_{k}^{T}
$$

and

$$
\begin{aligned}
\Sigma_{k+1 \mid k+1}= & \left(1+\theta_{3}+\theta_{4}\right)\left[\left(I-\sum_{i=1}^{N} H_{i} G_{k+1} T_{i} \bar{\Gamma}_{k+1} F_{k+1}\right)\left(\Sigma_{k+1 \mid k}^{-1}-\varepsilon_{2} I\right)^{-1}\right. \\
& \times\left(I-\sum_{i=1}^{N} H_{i} G_{k+1} T_{i} \bar{\Gamma}_{k+1} F_{k+1}\right)^{T}+\varepsilon_{2}^{-1}\left(\sum_{i=1}^{N} H_{i} G_{k+1} T_{i}\right) \bar{\Gamma}_{k+1} C_{k+1} \\
& \times C_{k+1}^{T} \bar{\Gamma}_{k+1}^{T}\left(\sum_{i=1}^{N} H_{i} G_{k+1} T_{i}\right)^{T}+\left(\sum_{i=1}^{N} H_{i} G_{k+1} T_{i}\right)\left(\breve{\Gamma}_{k+1} \circ V_{k+1}\right) \\
& \times\left(\sum_{i=1}^{N} H_{i} G_{k+1} T_{i}\right)^{T}+\left(1+\theta_{3}^{-1}+\theta_{5}\right) \rho^{2} \operatorname{tr}\left\{V_{k+1}\right\}\left(\sum_{i=1}^{N} H_{i} G_{k+1} T_{i}\right) \\
& \times\left(\sum_{i=1}^{N} H_{i} G_{k+1} T_{i}\right)^{T}+\left(\sum_{i=1}^{N} H_{i} G_{k+1} T_{i}\right)\left\{\left[\left(I-\underline{P}_{k+1}\right) \bar{Q}_{k+1}\right]\{I\right. \\
& \left.\left.-\left[\left(I-\bar{P}_{k+1}\right) \underline{Q}_{k+1}\right]^{T}\right\} \circ \bar{\Xi} I\right\}\left(\sum_{i=1}^{N} H_{i} G_{k+1} T_{i}\right)^{T}+\left(\sum_{i=1}^{N} H_{i} G_{k+1} T_{i}\right) \\
& \times\left(\bar{P}_{k+1} \circ \bar{Q}_{k+1} \bar{Q}_{k+1}^{T}\left(\sum_{i=1}^{N} H_{i} G_{k+1} T_{i}\right)^{+}+\left(1+\theta_{4}^{-1}+\theta_{5}^{-1}\right) \operatorname{tr}\left\{\left(I-\underline{P}_{k+1}\right)\right.\right. \\
& \left.\times \sum_{i=1}^{N} H_{i} G_{k+1} T_{i}\right)\left(\sum_{i=1}^{N} H_{i} G_{k+1} T_{i}\right)^{T}
\end{aligned}
$$

subject to two inequality conditions

$$
\begin{aligned}
& \varepsilon_{1}^{-1} I-\Sigma_{k-d \mid k-d}>0 \\
& \varepsilon_{2}^{-1} I-\Sigma_{k+1 \mid k}>0
\end{aligned}
$$

where

$$
\begin{aligned}
U_{k}= & \left(1+\theta_{1}^{-1}\right) \operatorname{tr}\left\{E_{k-d}\left(\Sigma_{k-d \mid k-d}^{-1}-\varepsilon_{1} I\right)^{-1} E_{k-d}^{T}+\varepsilon_{1}^{-1} B_{k-d} B_{k-d}^{T}\right\} \\
& +\left(1+\theta_{1}\right) \operatorname{tr}\left\{\bar{A}_{k} \Sigma_{k \mid k} \bar{A}_{k}^{T}\right\}, \quad \hbar=\sum_{i=1}^{n} s_{i, \max }^{2},
\end{aligned}
$$




$$
\begin{aligned}
& V_{k+1}= 2\left(\delta_{1}^{2} \operatorname{tr}\left\{\Phi_{k+1 \mid k}\right\}+\delta_{2}^{2}\right) I, \quad \rho=\max \left\{\rho_{i, k+1}\right\}, \quad \tilde{\rho}=\max \left\{\bar{\rho}_{i, k+1}\right\}, \\
& \bar{I}=\operatorname{col}_{N}\{I\}, \quad \Phi_{k+1 \mid k}=\left(1+\theta_{2}\right) \Sigma_{k+1 \mid k}+\left(1+\theta_{2}^{-1}\right) \hat{x}_{k+1 \mid k} \hat{x}_{k+1 \mid k}^{T}, \\
& \breve{\Gamma}_{k+1}=\operatorname{diag}\left\{\left(\bar{\gamma}_{1, k+1}+\rho\right)\left(1-\bar{\gamma}_{1, k+1}+\rho\right) I, \ldots,\left(\bar{\gamma}_{N, k+1}+\rho\right)\right. \\
&\left.\quad \times\left(1-\bar{\gamma}_{N, k+1}+\rho\right) I\right\}, \quad \underline{Q}_{k+1}=\bar{\Lambda}_{k+1}-\tilde{\rho} I, \\
& \bar{P}_{k+1}=\bar{\Gamma}_{k+1}+\rho I, \quad \underline{P}_{k+1}=\bar{\Gamma}_{k+1}-\rho I, \quad \bar{Q}_{k+1}=\bar{\Lambda}_{k+1}+\tilde{\rho} I,
\end{aligned}
$$

then, one can find an upper bound matrix, i.e.,

$$
P_{k+1 \mid k+1} \leq \Sigma_{k+1 \mid k+1}
$$

Proof Based on the property of trace and Lemma 3, the first two terms of (18) can be expressed as

$$
\mathbb{E}\left\{\Pi_{k} \bar{A}_{k} e_{k \mid k} e_{k \mid k}^{T} \bar{A}_{k}^{T} \Pi_{k}^{T}\right\} \leq \mathbb{E}\left\{\left\|\bar{A}_{k} e_{k \mid k}\right\|^{2}\right\} I=\operatorname{tr}\left\{\bar{A}_{k} P_{k \mid k} \bar{A}_{k}^{T}\right\} I
$$

and

$$
\begin{aligned}
\mathcal{A}_{k} & \leq \mathbb{E}\left\{\left\|\left(E_{k-d}+B_{k-d} \Theta_{k-d}\right) e_{k-d \mid k-d}\right\|^{2}\right\} I \\
& \leq \operatorname{tr}\left\{E_{k-d}\left(P_{k-d \mid k-d}^{-1}-\varepsilon_{1} I\right)^{-1} E_{k-d}^{T}+\varepsilon_{1}^{-1} B_{k-d} B_{k-d}^{T}\right\} I,
\end{aligned}
$$

where $\varepsilon_{1}$ is a positive scalar. By means of an elementary inequality, the cross-term $\mathcal{B}_{k}+\mathcal{B}_{k}^{T}$ in (18) can be handled as

$$
\mathcal{B}_{k}+\mathcal{B}_{k}^{T} \leq \theta_{1} \mathbb{E}\left\{\Pi_{k} \bar{A}_{k} e_{k \mid k} e_{k \mid k}^{T} \bar{A}_{k}^{T} \Pi_{k}^{T}\right\}+\theta_{1}^{-1} \mathcal{A}_{k},
$$

with $\theta_{1}$ being a positive constant. Furthermore, from the perspective of saturation level, the following relationship can be established:

$$
\begin{aligned}
P_{k+1 \mid k}=\mathbb{E}\left\{\left[\begin{array}{c}
\mu\left(A_{k} x_{k}+f\left(x_{k-d}\right)\right)-\mu\left(A_{k} \hat{x}_{1, k \mid k}+f\left(\hat{x}_{1, k-d \mid k-d}\right)\right) \\
\mu\left(A_{k} x_{k}+f\left(x_{k-d}\right)\right)-\mu\left(A_{k} \hat{x}_{2, k \mid k}+f\left(\hat{x}_{2, k-d \mid k-d}\right)\right) \\
\vdots \\
\mu\left(A_{k} x_{k}+f\left(x_{k-d}\right)\right)-\mu\left(A_{k} \hat{x}_{N, k \mid k}+f\left(\hat{x}_{N, k-d \mid k-d}\right)\right)
\end{array}\right]\right. \\
\left.\times\left[\begin{array}{c}
\mu\left(A_{k} x_{k}+f\left(x_{k-d}\right)\right)-\mu\left(A_{k} \hat{x}_{1, k \mid k}+f\left(\hat{x}_{1, k-d \mid k-d}\right)\right) \\
\mu\left(A_{k} x_{k}+f\left(x_{k-d}\right)\right)-\mu\left(A_{k} \hat{x}_{2, k \mid k}+f\left(\hat{x}_{2, k-d \mid k-d}\right)\right) \\
\vdots \\
\mu\left(A_{k} x_{k}+f\left(x_{k-d}\right)\right)-\mu\left(A_{k} \hat{x}_{N, k \mid k}+f\left(\hat{x}_{N, k-d \mid k-d}\right)\right)
\end{array}\right]\right\} \\
+\bar{B}_{k} R_{k} \bar{B}_{k}^{T} \\
\leq 4 N \hbar I+\bar{B}_{k} R_{k} \bar{B}_{k}^{T} .
\end{aligned}
$$

Substituting (25)-(28) into (18), one has

$$
P_{k+1 \mid k} \leq \min \left\{W_{k}, 4 N \hbar\right\} I+\bar{B}_{k} R_{k} \bar{B}_{k}^{T}
$$

with $W_{k}=\left(1+\theta_{1}\right) \operatorname{tr}\left\{\bar{A}_{k} P_{k \mid k} \bar{A}_{k}^{T}\right\}+\left(1+\theta_{1}^{-1}\right) \operatorname{tr}\left\{E_{k-d}\left(P_{k-d \mid k-d}^{-1}-\varepsilon_{1} I\right)^{-1} E_{k-d}^{T}+\varepsilon_{1}^{-1} B_{k-d} B_{k-d}^{T}\right\}$. 
In the sequel, we are ready to discuss the covariance matrix $P_{k+1 \mid k+1}$ and look for an upper bound. By virtue of Lemma 3, the first term of (19) can be bounded as

$$
\begin{aligned}
\mathbb{E}\left\{\left[I-\sum_{i=1}^{N} H_{i} G_{k+1} T_{i} \bar{\Gamma}_{k+1}\left(F_{k+1}+C_{k+1} \Omega_{k+1}\right)\right] e_{k+1 \mid k} e_{k+1 \mid k}^{T}\right. \\
\left.\quad \times\left[I-\sum_{i=1}^{N} H_{i} G_{k+1} T_{i} \bar{\Gamma}_{k+1}\left(F_{k+1}+C_{k+1} \Omega_{k+1}\right)\right]^{T}\right\} \\
\leq\left[\left(I-\sum_{i=1}^{N} H_{i} G_{k+1} T_{i} \bar{\Gamma}_{k+1} F_{k+1}\right)\left(P_{k+1 \mid k}^{-1}-\varepsilon_{2} I\right)^{-1}\left(I-\sum_{i=1}^{N} H_{i} G_{k+1} T_{i} \bar{\Gamma}_{k+1} F_{k+1}\right)^{T}\right] \\
\quad+\varepsilon_{2}^{-1}\left(\sum_{i=1}^{N} H_{i} G_{k+1} T_{i} \bar{\Gamma}_{k+1}\right) C_{k+1} C_{k+1}^{T}\left(\sum_{i=1}^{N} H_{i} G_{k+1} T_{i} \bar{\Gamma}_{k+1}\right)^{T}
\end{aligned}
$$

with $\varepsilon_{2}$ being a positive constant. Next, according to Lemma 2, we obtain

$$
\begin{aligned}
& \mathbb{E}\left\{\left(\sum_{i=1}^{N} H_{i} G_{k+1} T_{i}\right) \tilde{\Gamma}_{k+1} \bar{h}\left(x_{k+1}\right) \bar{h}^{T}\left(x_{k+1}\right) \tilde{\Gamma}_{k+1}^{T}\left(\sum_{i=1}^{N} H_{i} G_{k+1} T_{i}\right)^{T}\right\} \\
& =\left(\sum_{i=1}^{N} H_{i} G_{k+1} T_{i}\right)\left(\boldsymbol{\Gamma}_{k+1} \circ \mathbb{E}\left\{\bar{h}\left(x_{k+1}\right) \bar{h}^{T}\left(x_{k+1}\right)\right\}\right)\left(\sum_{i=1}^{N} H_{i} G_{k+1} T_{i}\right)^{T},
\end{aligned}
$$

where

$$
\begin{aligned}
\boldsymbol{\Gamma}_{k+1}= & \operatorname{diag}\left\{\left(\bar{\gamma}_{1, k+1}+\Delta \gamma_{1, k+1}\right)\left[1-\left(\bar{\gamma}_{1, k+1}+\Delta \gamma_{1, k+1}\right)\right] I,\right. \\
& \left(\bar{\gamma}_{2, k+1}+\Delta \gamma_{2, k+1}\right)\left[1-\left(\bar{\gamma}_{2, k+1}+\Delta \gamma_{2, k+1}\right)\right] I, \\
& \left.\ldots,\left(\bar{\gamma}_{N, k+1}+\Delta \gamma_{N, k+1}\right)\left[1-\left(\bar{\gamma}_{N, k+1}+\Delta \gamma_{N, k+1}\right)\right] I\right\} .
\end{aligned}
$$

Due to $\left|\Delta \gamma_{i, k}\right| \leq \rho_{i, k}$, we have

$$
\begin{aligned}
\boldsymbol{\Gamma}_{k+1} \leq & \operatorname{diag}\left\{\left(\bar{\gamma}_{1, k+1}+\rho_{1, k+1}\right)\left(1-\bar{\gamma}_{1, k+1}+\rho_{1, k+1}\right) I,\right. \\
& \left.\ldots,\left(\bar{\gamma}_{N, k+1}+\rho_{N, k+1}\right)\left(1-\bar{\gamma}_{N, k+1}+\rho_{N, k+1}\right) I\right\} \\
\leq & \breve{\Gamma}_{k+1}
\end{aligned}
$$

where $\breve{\Gamma}_{k+1}$ is given in (23). Additionally, it can be obtained from (3) that

$$
\begin{aligned}
\mathbb{E}\left\{\bar{h}\left(x_{k+1}\right) \bar{h}^{T}\left(x_{k+1}\right)\right\} & \leq \mathbb{E}\left\{\left(\delta_{1}\left\|\bar{x}_{k+1}\right\|+\delta_{2}\right)^{2}\right\} I \\
& \leq\left(2 \delta_{1}^{2} \mathbb{E}\left\{\left\|\bar{x}_{k+1}\right\|^{2}\right\}+2 \delta_{2}^{2}\right) I \\
& =2\left(\delta_{1}^{2} \operatorname{tr}\left\{\mathbb{E}\left\{\bar{x}_{k+1} \bar{x}_{k+1}^{T}\right\}\right\}+\delta_{2}^{2}\right) I .
\end{aligned}
$$

Using an elementary inequality, we have

$$
\mathbb{E}\left\{\bar{x}_{k+1} \bar{x}_{k+1}^{T}\right\} \leq \Psi_{k+1 \mid k}
$$


with $\Psi_{k+1 \mid k}=\left(1+\theta_{2}\right) P_{k+1 \mid k}+\left(1+\theta_{2}^{-1}\right) \hat{x}_{k+1 \mid k} \hat{x}_{k+1 \mid k}^{T}$ and $\theta_{2}$ being a positive constant. Besides, we have

$$
\mathbb{E}\left\{\tilde{\Gamma}_{k+1} \bar{h}\left(x_{k+1}\right) \bar{h}^{T}\left(x_{k+1}\right) \tilde{\Gamma}_{k+1}^{T}\right\} \leq \breve{\Gamma}_{k+1} \circ J_{k+1},
$$

where $J_{k+1}=2\left(\delta_{1}^{2} \operatorname{tr}\left\{\Psi_{k+1 \mid k}\right\}+\delta_{2}^{2}\right) I$. Thus, we have

$$
\begin{aligned}
\mathbb{E}\left\{\check{\Gamma}_{k+1} \bar{h}\left(x_{k+1}\right) \bar{h}^{T}\left(x_{k+1}\right) \check{\Gamma}_{k+1}^{T}\right\} & \leq \mathbb{E}\left\{\left\|\check{\Gamma}_{k+1} \bar{h}\left(x_{k+1}\right)\right\|^{2}\right\} I \\
& \leq \operatorname{tr}\left\{\check{\Gamma}_{k+1} J_{k+1} \check{\Gamma}_{k+1}^{T}\right\} I \\
& \leq \boldsymbol{\rho}^{2} \operatorname{tr}\left\{J_{k+1}\right\} I .
\end{aligned}
$$

From $\xi_{i, k+1}^{T} \xi_{i, k+1} \leq \bar{\xi}_{k+1}$, we get

$$
\Xi_{k+1} \Xi_{k+1}^{T} \leq\left\|\Xi_{k+1}\right\|^{2} I=\Xi_{k+1}^{T} \Xi_{k+1} I \leq \bar{\Xi} I
$$

Subsequently, one has

$$
\mathbb{E}\left\{\Xi_{k+1} \Xi_{k+1}^{T}\right\} \leq \bar{\Xi} I
$$

Therefore, the fourth term of (19) is estimated as follows:

$$
\begin{aligned}
\mathbb{E}\{ & \left(\sum_{i=1}^{N} H_{i} G_{k+1} T_{i}\right)\left[\left(I-\Gamma_{k+1}\right) \Lambda_{k+1}-\left(I-\hat{\Gamma}_{k+1}\right) \hat{\Lambda}_{k+1}\right] \Xi_{k+1} \\
& \left.\times \Xi_{k+1}^{T}\left[\left(I-\Gamma_{k+1}\right) \Lambda_{k+1}-\left(I-\hat{\Gamma}_{k+1}\right) \hat{\Lambda}_{k+1}\right]^{T}\left(\sum_{i=1}^{N} H_{i} G_{k+1} T_{i}\right)^{T}\right\} \\
\leq & \left(\sum_{i=1}^{N} H_{i} G_{k+1} T_{i}\right)\left\{\left[\left(I-\hat{\Gamma}_{k+1}\right) \hat{\Lambda}_{k+1}\right]\left\{I-\left[\left(I-\hat{\Gamma}_{k+1}\right) \hat{\Lambda}_{k+1}\right]\right\} \circ \bar{\Xi} I\right\} \\
& \times\left(\sum_{i=1}^{N} H_{i} G_{k+1} T_{i}\right)^{T} \\
\leq & \left(\sum_{i=1}^{N} H_{i} G_{k+1} T_{i}\right)\left\{\left(I-\underline{P}_{k+1}\right) \bar{Q}_{k+1}\left[I-\left(I-\bar{P}_{k+1}\right) \underline{Q}_{k+1}\right] \circ \bar{\Xi} I\right\} \\
& \times\left(\sum_{i=1}^{N} H_{i} G_{k+1} T_{i}\right)^{T} .
\end{aligned}
$$

Moreover, we get

$$
\mathbb{E}\left\{\Gamma_{k+1} v_{k+1} v_{k+1}^{T} \Gamma_{k+1}^{T}\right\} \leq \hat{\Gamma}_{k+1} \circ Q_{k+1} \leq \bar{P}_{k+1} \circ Q_{k+1} .
$$


The last quadratic term of (19) can be readily calculated as

$$
\begin{aligned}
\mathbb{E}\{[ & \left.\sum_{i=1}^{N} H_{i} G_{k+1} T_{i}\left(I-\hat{\Gamma}_{k+1}\right) \hat{\Lambda}_{k+1}\right] \Xi_{k+1} \Xi_{k+1}^{T} \\
& \left.\times\left[\sum_{i=1}^{N} H_{i} G_{k+1} T_{i}\left(I-\hat{\Gamma}_{k+1}\right) \hat{\Lambda}_{k+1}\right]^{T}\right\} \\
\leq & \left(\sum_{i=1}^{N} H_{i} G_{k+1} T_{i}\right) \mathbb{E}\left\{\left\|\left(I-\hat{\Gamma}_{k+1}\right) \hat{\Lambda}_{k+1} \Xi_{k+1}\right\|^{2}\right\}\left(\sum_{i=1}^{N} H_{i} G_{k+1} T_{i}\right)^{T} \\
\leq & \operatorname{tr}\left\{\left(I-\underline{P}_{k+1}\right) \bar{Q}_{k+1} \bar{\Xi} \bar{Q}_{k+1}^{T}\left(I-\underline{P}_{k+1}\right)^{T}\right\} \\
& \times\left(\sum_{i=1}^{N} H_{i} G_{k+1} T_{i}\right)\left(\sum_{i=1}^{N} H_{i} G_{k+1} T_{i}\right)^{T} .
\end{aligned}
$$

The relevant cross-terms in (19) are computed as follows:

$$
\begin{aligned}
-\mathcal{C}_{k+1}-\mathcal{C}_{k+1}^{T} \leq & \theta_{3} \mathbb{E}\left\{\left[I-\sum_{i=1}^{N} H_{i} G_{k+1} T_{i} \bar{\Gamma}_{k+1}\left(F_{k+1}+C_{k+1} \Omega_{k+1}\right)\right] e_{k+1 \mid k}\right. \\
& \left.\times e_{k+1 \mid k}^{T}\left[I-\sum_{i=1}^{N} H_{i} G_{k+1} T_{i} \bar{\Gamma}_{k+1}\left(F_{k+1} C_{k+1} \Omega_{k+1}\right)\right]^{T}\right\} \\
& +\theta_{3}^{-1} \mathbb{E}\left\{\left(\sum_{i=1}^{N} H_{i} G_{k+1} T_{i} \check{\Gamma}_{k+1}\right) \bar{h}\left(x_{k+1}\right) \bar{h}^{T}\left(x_{k+1}\right)\right. \\
& \left.\times\left(\sum_{i=1}^{N} H_{i} G_{k+1} T_{i} \check{\Gamma}_{k+1}\right)^{T}\right\}, \\
-\mathcal{D}_{k+1}-\mathcal{D}_{k+1}^{T} \leq & \theta_{4} \mathbb{E}\left\{\left[I-\sum_{i=1}^{N} H_{i} G_{k+1} T_{i} \bar{\Gamma}_{k+1}\left(F_{k+1}+C_{k+1} \Omega_{k+1}\right)\right] e_{k+1 \mid k}\right. \\
& \left.\times e_{k+1 \mid k}^{T}\left[I-\sum_{i=1}^{N} H_{i} G_{k+1} T_{i} \bar{\Gamma}_{k+1}\left(F_{k+1}+C_{k+1} \Omega_{k+1}\right)\right]^{T}\right\} \\
& +\theta_{4}^{-1} \mathbb{E}\left\{\left[\sum_{i=1}^{N} H_{i} G_{k+1} T_{i}\left(I-\hat{\Gamma}_{k+1}\right) \hat{\Lambda}_{k+1}\right] \Xi_{k+1} \Xi_{k+1}^{T}\right. \\
& \left.\times\left[\sum_{i=1}^{N} H_{i} G_{k+1} T_{i}\left(I-\hat{\Gamma}_{k+1}\right) \hat{\Lambda}_{k+1}\right]^{T}\right\}, \\
\mathcal{E}_{k+1}+\mathcal{E}_{k+1}^{T} \leq \theta_{5} \mathbb{E}\left\{\left(\sum_{i=1}^{N} H_{i} G_{k+1} T_{i} \check{\Gamma}_{k+1}\right) \bar{h}_{k+1}\left(x_{k+1}\right)\right. & \left.\left(\sum_{i=1}^{N} H_{i} G_{k+1} T_{i} \check{\Gamma}_{k+1}\right){ }^{T}\right\} \\
& \\
&
\end{aligned}
$$




$$
\begin{aligned}
& +\theta_{5}^{-1} \mathbb{E}\left\{\left[\sum_{i=1}^{N} H_{i} G_{k+1} T_{i}\left(I-\hat{\Gamma}_{k+1}\right) \hat{\Lambda}_{k+1}\right] \Xi_{k+1}\right. \\
& \left.\times \Xi_{k+1}^{T}\left[\sum_{i=1}^{N} H_{i} G_{k+1} T_{i}\left(I-\hat{\Gamma}_{k+1}\right) \hat{\Lambda}_{k+1}\right]^{T}\right\}
\end{aligned}
$$

where $\theta_{3}, \theta_{4}$ and $\theta_{5}$ are positive scalars. Consequently, we arrive at

$$
\begin{aligned}
P_{k+1 \mid k+1} & \\
\leq & \left(1+\theta_{3}+\theta_{4}\right)\left[\left(I-\sum_{i=1}^{N} H_{i} G_{k+1} T_{i} \bar{\Gamma}_{k+1} F_{k+1}\right)\left(P_{k+1 \mid k}^{-1}-\varepsilon_{2} I\right)^{-1}\right. \\
& \times\left(I-\sum_{i=1}^{N} H_{i} G_{k+1} T_{i} \bar{\Gamma}_{k+1} F_{k+1}\right)^{T}+\varepsilon_{2}^{-1}\left(\sum_{i=1}^{N} H_{i} G_{k+1} T_{i}\right) \bar{\Gamma}_{k+1} C_{k+1} \\
& \left.\times C_{k+1}^{T} \bar{\Gamma}_{k+1}^{T}\left(\sum_{i=1}^{N} H_{i} G_{k+1} T_{i}\right)^{T}\right]+\left(\sum_{i=1}^{N} H_{i} G_{k+1} T_{i}\right)\left(\breve{\Gamma}_{k+1} \circ J_{k+1}\right) \\
& \times\left(\sum_{i=1}^{N} H_{i} G_{k+1} T_{i}\right)^{T}+\left(1+\theta_{3}^{-1}+\theta_{5}\right) \rho^{2} \operatorname{tr}\left\{J_{k+1}\right\}\left(\sum_{i=1}^{N} H_{i} G_{k+1} T_{i}\right) \\
& \times\left(\sum_{i=1}^{N} H_{i} G_{k+1} T_{i}\right)^{T}+\left(\sum_{i=1}^{N} H_{i} G_{k+1} T_{i}\right)\left\{\left[\left(I-\underline{P}_{k+1} \bar{Q}_{k+1}\right]\{I\right.\right. \\
& \left.\left.-\left[\left(I-\bar{P}_{k+1}\right) \underline{Q}_{k+1}\right]^{T}\right\} \circ \bar{\Xi} I\right\}\left(\sum_{i=1}^{N} H_{i} G_{k+1} T_{i}\right)^{T}+\left(\sum_{i=1}^{N} H_{i} G_{k+1} T_{i}\right) \\
& \times\left(\bar{P}_{k+1} \circ Q_{k+1}\right)\left(\sum_{i=1}^{N} H_{i} G_{k+1} T_{i}\right)^{T}+\left(1+\theta_{4}^{-1}+\theta_{5}^{-1}\right) \operatorname{tr}\left\{\left(I-\underline{P}_{k+1}\right)\right. \\
& \left.\times \bar{Q}_{k+1} \bar{\Xi} \bar{Q}_{k+1}^{T}\left(I-\underline{P}_{k+1}\right)^{T}\right\}\left(\sum_{i=1}^{N} H_{i} G_{k+1} T_{i}\right)\left(\sum_{i=1}^{N} H_{i} G_{k+1} T_{i}\right)^{T} .
\end{aligned}
$$

From (20), (23), and (29), we know $P_{k+1 \mid k} \leq \Sigma_{k+1 \mid k}$. Thus, one has

$$
\left(P_{k+1 \mid k}^{-1}-\varepsilon_{2} I\right)^{-1} \leq\left(\Sigma_{k+1 \mid k}^{-1}-\varepsilon_{2} I\right)^{-1}
$$

Next, in terms of (43), (44), and by utilizing the mathematical induction method, we have $P_{k+1 \mid k+1} \leq \Sigma_{k+1 \mid k+1}$, which ends the proof of Theorem 1 .

Remark 3 In Theorem 1, the upper bound has been constructed based on the given $G_{k+1}$. Subsequently, we aim to choose a suitable filter parameter $G_{k+1}$ in order to optimize such an upper bound. However, it should be noted that the sensors only transmit information with their neighbors, which causes the matrix $T_{i}$ to be noninvertible. Hence, it induces some difficulties for the later filtering developments. Here, an MST is adopted to cope with this problem. 
To proceed, some notations are introduced, which are necessary to the subsequent derivations:

$$
\begin{aligned}
\mathcal{O}_{k+1}= & \left(1+\theta_{3}+\theta_{4}\right)\left(\Sigma_{k+1 \mid k}^{-1}-\varepsilon_{2} I\right)^{-1} F_{k+1}^{T} \bar{\Gamma}_{k+1}^{T}=\left[\mathcal{O}_{k+1}^{(i)}\right]_{N \times 1}, \\
\bar{G}_{k+1}^{(i)}= & \mathcal{O}_{k+1}^{(i)} \bar{L}_{i}\left(\bar{L}_{i}^{T} \mathcal{L}_{k+1} \bar{L}_{i}\right)^{-1} \bar{L}_{i}^{T}, \quad L_{i}=\operatorname{diag}\left\{\sqrt{\alpha_{i j}} I\right\}, \\
\mathcal{L}_{k+1}= & \left(1+\theta_{3}+\theta_{4}\right) \bar{\Gamma}_{k+1} F_{k+1}\left(\Sigma_{k+1 \mid k}^{-1}-\varepsilon_{2} I\right)^{-1} F_{k+1}^{T} \bar{\Gamma}_{k+1}^{T} \\
& +\left(1+\theta_{3}+\theta_{4}\right) \varepsilon_{2}^{-1} \bar{\Gamma}_{k+1} C_{k+1} C_{k+1}^{T} \bar{\Gamma}_{k+1}^{T}+\bar{P}_{k+1} \circ Q_{k+1} \\
& +\breve{\Gamma}_{k+1} \circ V_{k+1}+\left(1+\theta_{3}^{-1}+\theta_{5}\right) \rho^{2} \operatorname{tr}\left\{V_{k+1}\right\} I \\
& +\left[\left(I-\underline{P}_{k+1}\right) \bar{Q}_{k+1}\right]\left\{I-\left[\left(I-\bar{P}_{k+1}\right) \underline{Q}_{k+1}\right]^{T}\right\} \circ \bar{\Xi} I \\
& +\left(1+\theta_{4}^{-1}+\theta_{5}^{-1}\right) \operatorname{tr}\left\{\left(I-\underline{P}_{k+1}\right) \bar{Q}_{k+1} \bar{\Xi} \bar{Q}_{k+1}^{T}\left(I-\underline{P}_{k+1}\right)^{T}\right\} I, \\
G_{k+1}^{(i)}= & {\left[G_{i j, k+1}\right]_{1 \times N}, \quad \bar{G}_{k+1}^{(i)}=\left[\bar{G}_{i j, k+1}\right]_{1 \times N}, \quad G_{k+1}=\left[G_{k+1}^{(i)}\right]_{N \times 1} . }
\end{aligned}
$$

In what follows, we aim to provide the design approach of filter parameter by using the MST and optimize the given upper bound accordingly.

Theorem 2 Consider the filter (9) and (10). Then $\operatorname{tr}\left\{\Sigma_{k+1 \mid k+1}\right\}$ is minimized by utilizing the following filter parameter:

$$
G_{i j, k+1}= \begin{cases}\bar{G}_{i j, k+1} \alpha_{i j}^{-1} & \text { if } \alpha_{i j} \neq 0, \\ 0 & \text { otherwise. }\end{cases}
$$

Proof In light of (21) and the following equality

$$
\operatorname{tr}\left\{H_{i} M H_{j}^{T}\right\}=\operatorname{tr}\left\{H_{j}^{T} H_{i} M\right\}=0,
$$

then we obtain

$$
\begin{aligned}
\operatorname{tr}\left\{\Sigma_{k+1 \mid k+1}\right\} & \left(1+\theta_{3}+\theta_{4}\right) \operatorname{tr}\left\{\left(\Sigma_{k+1 \mid k}^{-1}-\varepsilon_{2} I\right)^{-1}-\sum_{i=1}^{N} H_{i} G_{k+1} T_{i} \bar{\Gamma}_{k+1} F_{k+1}\right. \\
& \times\left(\Sigma_{k+1 \mid k}^{-1}-\varepsilon_{2} I\right)^{-1}-\left(\Sigma_{k+1 \mid k}^{-1}-\varepsilon_{2} I\right)^{-1}\left(\sum_{i=1}^{N} H_{i} G_{k+1} T_{i} \bar{\Gamma}_{k+1} F_{k+1}\right)^{T} \\
& +\sum_{i=1}^{N}\left(H_{i} G_{k+1} T_{i} \bar{\Gamma}_{k+1} F_{k+1}\right)\left(\Sigma_{k+1 \mid k}^{-1}-\varepsilon_{2} I\right)^{-1}\left(H_{i} G_{k+1} T_{i} \bar{\Gamma}_{k+1} F_{k+1}\right)^{T} \\
& \left.+\varepsilon_{2}^{-1} \sum_{i=1}^{N}\left(H_{i} G_{k+1} T_{i} \bar{\Gamma}_{k+1}\right) C_{k+1} C_{k+1}^{T}\left(H_{i} G_{k+1} T_{i} \bar{\Gamma}_{k+1}\right)^{T}\right\} \\
& +\operatorname{tr}\left\{\sum_{i=1}^{N}\left(H_{i} G_{k+1} T_{i}\right)\left(\breve{\Gamma}_{k+1} \circ V_{k+1}\right)\left(H_{i} G_{k+1} T_{i}\right)^{T}\right\}+\left(1+\theta_{3}^{-1}+\theta_{5}\right) \\
& \times \operatorname{tr}\left\{\sum_{i=1}^{N}\left(H_{i} G_{k+1} T_{i}\right) \rho^{2} \operatorname{tr}\left\{V_{k+1}\right\}\left(H_{i} G_{k+1} T_{i}\right)^{T}\right\}+\operatorname{tr}\left\{\sum_{i=1}^{N}\left(H_{i} G_{k+1} T_{i}\right)\right.
\end{aligned}
$$




$$
\begin{aligned}
& \left.\times\left\{\left[\left(I-\underline{P}_{k+1}\right) \bar{Q}_{k+1}\right]\left[I-\left[\left(I-\bar{P}_{k+1}\right) \underline{Q}_{k+1}\right]^{T}\right] \circ \bar{\Xi} I\right\}\left(H_{i} G_{k+1} T_{i}\right)^{T}\right\} \\
& +\operatorname{tr}\left\{\sum_{i=1}^{N}\left(H_{i} G_{k+1} T_{i}\right)\left(\bar{P}_{k+1} \circ Q_{k+1}\right)\left(H_{i} G_{k+1} T_{i}\right)^{T}\right\}+\left(1+\theta_{4}^{-1}+\theta_{5}^{-1}\right) \\
& \times \operatorname{tr}\left\{\sum_{i=1}^{N}\left(H_{i} G_{k+1} T_{i}\right) \operatorname{tr}\left\{\left(I-\underline{P}_{k+1}\right) \bar{Q}_{k+1} \overline{\bar{\Xi}} \bar{Q}_{k+1}^{T}\left(I-\underline{P}_{k+1}\right)^{T}\right\}\right. \\
& \left.\times\left(H_{i} G_{k+1} T_{i}\right)^{T}\right\} .
\end{aligned}
$$

Subsequently, we aim to derive the expression form of the filter parameter $G_{k+1}$. In particular, take the partial derivative of $\operatorname{tr}\left\{\Sigma_{k+1 \mid k+1}\right\}$ with respect to $G_{k+1}$. By setting the result be zero, the following equation can be obtained:

$$
\begin{aligned}
\frac{\partial \operatorname{tr}\left\{\Sigma_{k+1 \mid k+1}\right\}}{\partial G_{k+1}} & -2\left(1+\theta_{3}+\theta_{4}\right) \sum_{i=1}^{N} H_{i}\left(\Sigma_{k+1 \mid k}^{-1}-\varepsilon_{2} I\right)^{-1} F_{k+1}^{T} \bar{\Gamma}_{k+1}^{T} T_{i}^{T}+2\left(1+\theta_{3}+\theta_{4}\right) \sum_{i=1}^{N} H_{i} \\
& \times G_{k+1} T_{i} \bar{\Gamma}_{k+1} F_{k+1}\left(\Sigma_{k+1 \mid k}^{-1}-\varepsilon_{2} I\right)^{-1} F_{k+1}^{T} \bar{\Gamma}_{k+1}^{T} T_{i}^{T}+2\left(1+\theta_{3}+\theta_{4}\right) \varepsilon_{2}^{-1} \\
& \times \sum_{i=1}^{N} H_{i} G_{k+1} T_{i} \bar{\Gamma}_{k+1} C_{k+1} C_{k+1}^{T} \bar{\Gamma}_{k+1}^{T} T_{i}^{T}+2 \sum_{i=1}^{N} H_{i} G_{k+1} T_{i}\left(\breve{\Gamma}_{k+1} \circ V_{k+1}\right) T_{i}^{T} \\
& +2\left(1+\theta_{3}^{-1}+\theta_{5}\right) \rho^{2} \sum_{i=1}^{N} H_{i} G_{k+1} T_{i} \operatorname{tr}\left\{V_{k+1}\right\} T_{i}^{T}+2 \sum_{i=1}^{N} H_{i} G_{k+1} T_{i}\left\{\left[\left(I-\underline{P}_{k+1}\right)\right.\right. \\
& \left.\left.\times \bar{Q}_{k+1}\right]\left\{I-\left[\left(I-\bar{P}_{k+1}\right) \underline{Q}_{k+1}\right]^{T}\right\} \circ \bar{\Xi} I\right\} T_{i}^{T}+2 \sum_{i=1}^{N} H_{i} G_{k+1} T_{i}\left(\bar{P}_{k+1} \circ Q_{k+1}\right) T_{i}^{T} \\
& +2\left(1+\theta_{4}^{-1}+\theta_{5}^{-1}\right) \sum_{i=1}^{N} H_{i} G_{k+1} T_{i} \operatorname{tr}\left\{\left(I-\underline{P}_{k+1}\right) \bar{Q}_{k+1} \bar{\Xi} \bar{Q}_{k+1}^{T}\left(I-\underline{P}_{k+1}\right)^{T}\right\} T_{i}^{T} \\
= & 2 \sum_{i=1}^{N} H_{i} G_{k+1} T_{i} \mathcal{L}_{k+1} T_{i}^{T}-2 \sum_{i=1}^{N} H_{i} \mathcal{O}_{k+1} T_{i}^{T} \\
= & 0 .
\end{aligned}
$$

On the basis of (49), we have

$$
G_{k+1}^{(i)} T_{i} \mathcal{L}_{k+1} T_{i}^{T}=\mathcal{O}_{k+1}^{(i)} T_{i}^{T}
$$

Noting $T_{i}=\bar{L}_{i} \bar{L}_{i}^{T}$, we arrive at

$$
G_{k+1}^{(i)} \bar{L}_{i} \bar{L}_{i}^{T} \mathcal{L}_{k+1} \bar{L}_{i} \bar{L}_{i}^{T}=\mathcal{O}_{k+1}^{(i)} \bar{L}_{i} \bar{L}_{i}^{T} .
$$

We know that $\bar{L}_{i}^{T}$ has full row rank and $\bar{L}_{i}^{T} \mathcal{L}_{k+1} \bar{L}_{i}$ is invertible, thus it can be derived that

$$
G_{k+1}^{(i)} \bar{L}_{i}=\mathcal{O}_{k+1}^{(i)} \bar{L}_{i}\left(\bar{L}_{i}^{T} \mathcal{L}_{k+1} \bar{L}_{i}\right)^{-1} .
$$


According to (45) and (52), one has

$$
\bar{G}_{k+1}^{(i)}=G_{k+1}^{(i)} T_{i}
$$

and then the filter parameter can be computed by:

$$
G_{i j, k+1}= \begin{cases}\bar{G}_{i j, k+1} \alpha_{i j}^{-1}, & j \in \mathcal{N}_{i}, \\ 0, & j \notin \mathcal{N}_{i},\end{cases}
$$

which ends the proof of Theorem 2.

\subsection{Error boundedness discussion}

In the subsection, the boundedness analysis regarding the trace of the upper bound for filtering error covariance is conducted. Before further developments, the following assumption is essential to be introduced.

Assumption 1 For the matrices $B_{k}$ and $R_{k}$, there exist a scalar $\vartheta>0$ and a positive semidefinite matrix $R \in \mathbb{R}^{n_{\omega} \times n_{\omega}}$ such that $\sigma_{\max }\left(B_{k}\right) \leq \vartheta$ and $R_{k} \leq R$ hold.

Theorem 3 For the SSNSs (1), (2), and (6) with (9)-(10), it follows from Assumption 1 that $\operatorname{tr}\left\{\Sigma_{k+1 \mid k+1}\right\}$ is bounded.

Proof Based on (45) and (48), one gets

$$
\begin{aligned}
& \operatorname{tr}\left\{\Sigma_{k+1 \mid k+1}\right\} \\
& =\operatorname{tr}\left\{\sum_{i=1}^{N}\left(H_{i} G_{k+1} T_{i}\right) \mathcal{L}_{k+1}\left(H_{i} G_{k+1} T_{i}\right)^{T}\right\}-\left(1+\theta_{3}+\theta_{4}\right) \operatorname{tr}\left\{\sum_{i=1}^{N} H_{i} G_{k+1} T_{i}\right. \\
& \quad \times \bar{\Gamma}_{k+1} F_{k+1}\left(\Sigma_{k+1 \mid k}^{-1}-\varepsilon_{2} I\right)^{-1}+\left(\Sigma_{k+1 \mid k}^{-1}-\varepsilon_{2} I\right)^{-1}\left(\sum_{i=1}^{N} H_{i} G_{k+1} T_{i} \bar{\Gamma}_{k+1} F_{k+1}\right)^{T} \\
& \left.\quad-\left(\Sigma_{k+1 \mid k}^{-1}-\varepsilon_{2} I\right)^{-1}\right\}
\end{aligned}
$$

For the first term of (55), one has

$$
\begin{aligned}
& \operatorname{tr}\left\{\sum_{i=1}^{N}\left(H_{i} G_{k+1} T_{i}\right) \mathcal{L}_{k+1}\left(H_{i} G_{k+1} T_{i}\right)^{T}\right\} \\
& \quad=\operatorname{tr}\left\{\sum_{i=1}^{N} H_{i} \mathcal{O}_{k+1}\left(H_{i} G_{k+1} T_{i}\right)^{T}\right\} \\
& \quad=\left(1+\theta_{3}+\theta_{4}\right) \operatorname{tr}\left\{\left(\Sigma_{k+1 \mid k}^{-1}-\varepsilon_{2} I\right)^{-1} F_{k+1}^{T} \bar{\Gamma}_{k+1}^{T}\left(\sum_{i=1}^{N} H_{i} G_{k+1} T_{i}\right)^{T}\right\} .
\end{aligned}
$$


Substituting (56) into (55) leads to

$$
\begin{aligned}
& \operatorname{tr}\left\{\Sigma_{k+1 \mid k+1}\right\} \\
& =-\left(1+\theta_{3}+\theta_{4}\right) \operatorname{tr}\left\{\sum_{i=1}^{N} H_{i} G_{k+1} T_{i} \bar{\Gamma}_{k+1} F_{k+1}\left(\Sigma_{k+1 \mid k}^{-1}-\varepsilon_{2} I\right)^{-1}\right\} \\
& +\left(1+\theta_{3}+\theta_{4}\right) \operatorname{tr}\left\{\left(\Sigma_{k+1 \mid k}^{-1}-\varepsilon_{2} I\right)^{-1}\right\} \\
& =-\left(1+\theta_{3}+\theta_{4}\right) \operatorname{tr}\left\{\sum_{i=1}^{N} H_{i} \mathcal{O}_{k+1} \bar{L}_{i}\left(\bar{L}_{i}^{T} \mathcal{L}_{k+1} \bar{L}_{i}\right)^{-1} \bar{L}_{i}^{T} \bar{\Gamma}_{k+1} F_{k+1}\right. \\
& \left.\quad \times\left(\Sigma_{k+1 \mid k}^{-1}-\varepsilon_{2} I\right)^{-1}\right\}+\left(1+\theta_{3}+\theta_{4}\right) \operatorname{tr}\left\{\left(\Sigma_{k+1 \mid k}^{-1}-\varepsilon_{2} I\right)^{-1}\right\} .
\end{aligned}
$$

In view of (20), we have

$$
\operatorname{tr}\left\{\Sigma_{k+1 \mid k}\right\} \leq 4 n N^{2} \hbar+\operatorname{tr}\left\{\bar{B}_{k} R_{k} \bar{B}_{k}^{T}\right\}=4 n N^{2} \hbar+\operatorname{tr}\left\{\bar{B}_{k}^{T} \bar{B}_{k} R_{k}\right\}
$$

In particular, by considering the properties of the trace and Assumption 1, one gets

$$
\operatorname{tr}\left\{\bar{B}_{k}^{T} \bar{B}_{k} R_{k}\right\} \leq \rho\left(\bar{B}_{k}^{T} \bar{B}_{k}\right) \operatorname{tr}\left\{R_{k}\right\} \leq N \vartheta^{2} \operatorname{tr}\{R\}
$$

and then

$$
\operatorname{tr}\left\{\Sigma_{k+1 \mid k}\right\} \leq 4 n N^{2} \hbar+N \vartheta^{2} \operatorname{tr}\{R\}
$$

On the other hand, it is not difficult to check that

$$
\Sigma_{k+1 \mid k} \leq \lambda_{\max }\left(\Sigma_{k+1 \mid k}\right) I \leq \operatorname{tr}\left\{\Sigma_{k+1 \mid k}\right\} I
$$

and further, one has

$$
\Sigma_{k+1 \mid k}^{-1}-\varepsilon_{2} I \geq\left(\frac{1}{\operatorname{tr}\left\{\Sigma_{k+1 \mid k}\right\}}-\varepsilon_{2}\right) I .
$$

Next, in view of the matrix operations, the following inequality can be written:

$$
\left(\Sigma_{k+1 \mid k}^{-1}-\varepsilon_{2} I\right)^{-1} \leq \frac{1}{\frac{1}{\operatorname{tr}\left\{\Sigma_{k+1 \mid k}\right\}}-\varepsilon_{2}} I .
$$

For the second term of (57), combining (60) with (63), we arrive at

$$
\begin{aligned}
& \left(1+\theta_{3}+\theta_{4}\right) \operatorname{tr}\left\{\left(\Sigma_{k+1 \mid k}^{-1}-\varepsilon_{2} I\right)^{-1}\right\} \\
& \leq\left(1+\theta_{3}+\theta_{4}\right) \operatorname{tr}\left\{\frac{1}{\frac{1}{4 n N^{2} \hbar+N \vartheta^{2} \operatorname{tr}\{R\}}-\varepsilon_{2}} I\right\} .
\end{aligned}
$$

Finally, it can be observed that the second term of (57) is bounded and the first term is nonpositive. Thereby, it can be shown that $\operatorname{tr}\left\{\Sigma_{k+1 \mid k+1}\right\}$ is bounded, which finishes the proof of Theorem 3. 
Remark 4 So far, the DEKF issue has been handled for SSNSs subject to ROCAs with UOPs. In particular, a novel DEKF algorithm has been established to analyze the impact of ROCAs with UOPs, and a recursive filtering scheme has been given to choose the filter parameter matrix. Furthermore, the boundedness of the estimation performance has been discussed, which further clarifies the performance analysis of the proposed filtering method from the theoretical viewpoint. It should be noticed that the proposed filtering method has the time-varying distributed manner, which has a potential advantage in the online implementation/application. Moreover, it is worthwhile to mention that some interesting and effective methods have been given in [37-42] for fractional systems, which motivate the further investigation on the DF problem for fractional nonlinear systems under ROCAs.

\section{Two illustrative simulations}

In this section, we will present two examples and discuss the effectiveness of the proposed DEKF scheme. In particular, comparative experiments for different cases are provided.

Example 1 Consider the SSNSs (1) with state delay $d=1$ and real measurement output (6). The related system parameters are given as:

$$
A_{k}=\left[\begin{array}{cc}
0.15+0.45 \sin (0.1 k) & -1.7-0.2 \cos (0.98 k) \\
0.05 & -0.3-0.65 \cos (0.7 k)
\end{array}\right], \quad B_{k}=\left[\begin{array}{c}
0.3 \\
0.35
\end{array}\right] \text {. }
$$

The WSN depicted in Fig. 2 is characterized by $\mathcal{G}=(\mathcal{U}, \mathcal{E}, \mathcal{A})$ with $\mathcal{U}=\{1,2,3,4\}, \mathcal{E}=$ $\{(1,1),(1,2),(1,4),(2,2),(2,3),(3,1),(3,2),(3,3),(4,3),(4,4)\}$ and

$$
\mathcal{A}=\left[\begin{array}{llll}
1 & 1 & 0 & 1 \\
0 & 1 & 1 & 0 \\
1 & 1 & 1 & 0 \\
0 & 0 & 1 & 1
\end{array}\right] .
$$

The nonlinear function $f\left(x_{k-d}\right)$ with state delay is expressed as

$$
f\left(x_{k-d}\right)=\left[\begin{array}{c}
0.12-2.15 \cos \left(x_{2, k-d}\right) \\
0.24 x_{1, k-d}-1.15 \sin \left(x_{2, k-d}\right)
\end{array}\right] \text {. }
$$

The nonlinear functions $h_{i}\left(x_{k}\right)$ are given by:

$$
\begin{aligned}
& h_{1}\left(x_{k}\right)=2.4+0.1 \sin \left(x_{1, k}\right), \\
& h_{2}\left(x_{k}\right)=5.6+0.1 \cos \left(x_{2, k}\right),
\end{aligned}
$$

Figure 2 The topological graph of WSNs

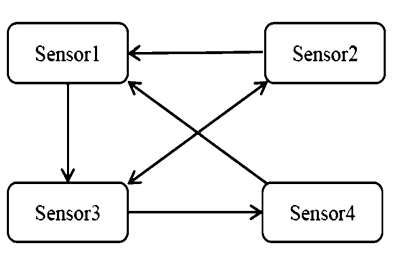




$$
\begin{aligned}
& h_{3}\left(x_{k}\right)=1.7+0.1 \cos \left(x_{1, k}\right), \\
& h_{4}\left(x_{k}\right)=5.1+0.1 \sin \left(x_{2, k}\right) .
\end{aligned}
$$

The covariance of process noise is $R_{k}=0.25$. The covariances of the measurement noises are $Q_{i, k}=0.15(i=1,2,3,4)$. Other parameters are chosen as $\varsigma_{1, \max }=6, \varsigma_{2, \max }=4$, $\theta_{1}=\theta_{3}=\theta_{4}=\theta_{5}=0.1, \theta_{2}=1.5, \bar{\Xi}=2, \bar{\gamma}_{i, k}=0.5, \bar{\lambda}_{i, k}=0.55, \rho=\tilde{\rho}=0.01, \delta_{1}=0.17$, $\delta_{2}=5, B_{i, k-d}=\operatorname{diag}\{0.1,0.25\}$, and $C_{j, k+1}=\operatorname{diag}\{0.1,0.2\}$. The initial values are set as $\bar{x}_{0}=\left[\begin{array}{ll}0 & 0\end{array}\right]^{T}, P_{0}=\operatorname{diag}\{2,2\}, \bar{x}_{-1}=\left[\begin{array}{ll}0 & 0\end{array}\right]^{T}, P_{-1}=\operatorname{diag}\{1.5,2\}, \hat{x}_{1,0 \mid 0}=\hat{x}_{1,-1 \mid-1}=\left[\begin{array}{ll}-2.8 & 2.5\end{array}\right]^{T}$, $\hat{x}_{2,0 \mid 0}=\hat{x}_{2,-1 \mid-1}=\left[\begin{array}{ll}-2.3 & 2.2\end{array}\right]^{T}, \hat{x}_{3,0 \mid 0}=\hat{x}_{3,-1 \mid-1}=\left[\begin{array}{ll}-2.3 & 2.3\end{array}\right]^{T}, \hat{x}_{4,0 \mid 0}=\hat{x}_{4,-1 \mid-1}=\left[\begin{array}{ll}-2.2 & 2.5\end{array}\right]^{T}$, $\Sigma_{1,0 \mid 0}=\operatorname{diag}\{15,15\}, \Sigma_{2,0 \mid 0}=\operatorname{diag}\{20,20\}, \Sigma_{3,0 \mid 0}=\operatorname{diag}\{25,25\}$, and $\Sigma_{4,0 \mid 0}=\operatorname{diag}\{30,30\}$. Based on (20)-(21) and (46), the optimal upper bound can be obtained at each sampling time.

In order to evaluate the algorithm accuracy of the new DEKF strategy, the mean square error (MSE) is utilized, which can be calculated by

$$
\operatorname{MSE}_{k}=\frac{1}{M} \mathbb{E}\left\{\sum_{\ell=1}^{M}\left(x_{k}^{(\ell)}-\hat{x}_{k \mid k}^{(\ell)}\right)^{T}\left(x_{k}^{(\ell)}-\hat{x}_{k \mid k}^{(\ell)}\right)\right\} .
$$

Here, $M$ represents the experimental number of runs. Accordingly, the main results are proposed in Figs. 3-7 with $M=200$. In particular, Figs. 3-4 show the trajectories of the estimates and the actual state $x_{k}$. We discover that the MSE is indeed below the upper bound in Fig. 5. Figure 6 exhibits the successful time of cyberattacks in detail. Figure 7 describes the curves of the upper bound affected by the different occurrence probabilities of cyberattacks, and it can be easily seen that the upper bound will increase when the occurring probabilities of cyberattacks increase. To sum up, those above simulation results show that the designed filtering strategy possesses good performance for addressed SSNSs under ROCAs with UOPs.

Next, we consider a 3-dimensional system in order to propose a comparative experiment and further testify the validity of the proposed DEKF algorithm.

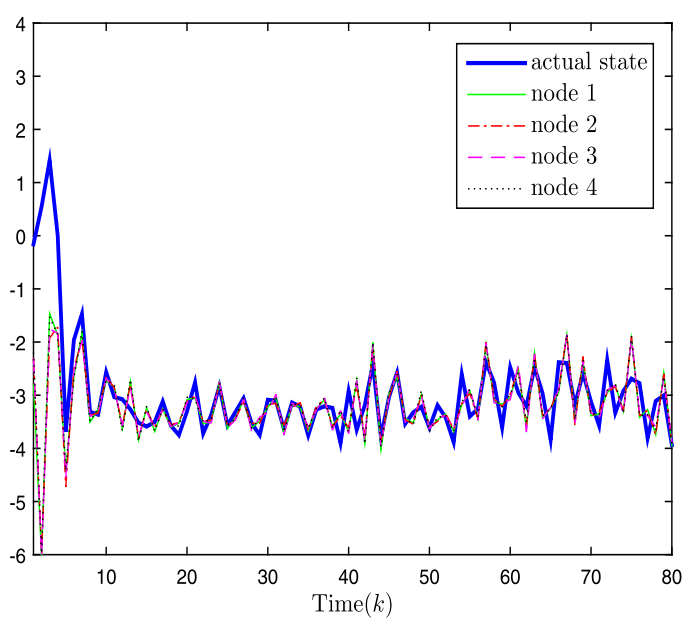

Figure 3 The actual state $x_{1, k}$ and the corresponding estimates 


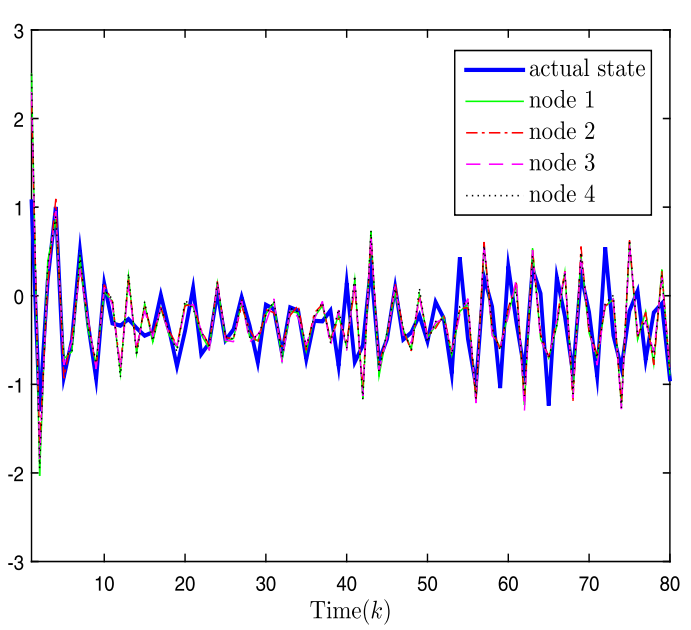

Figure 4 The actual state $x_{2, k}$ and the corresponding estimates

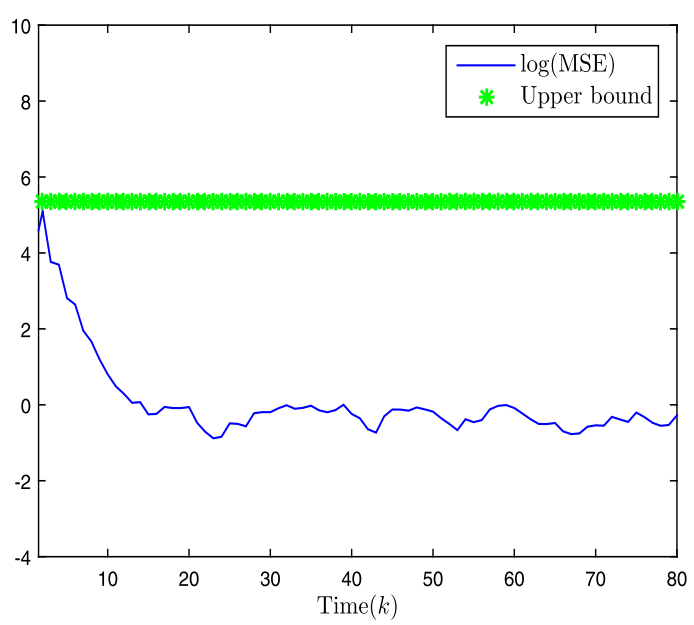

Figure 5 Log(MSE) and the upper bound

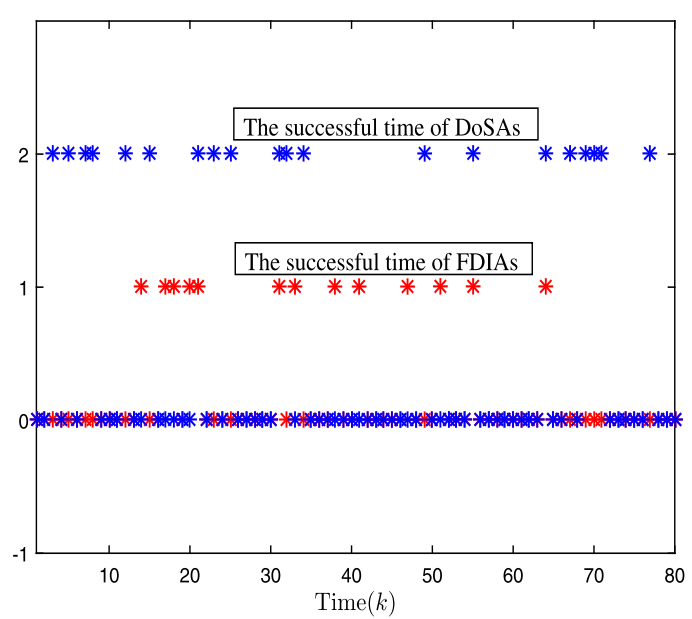

Figure 6 The successful attacks time 


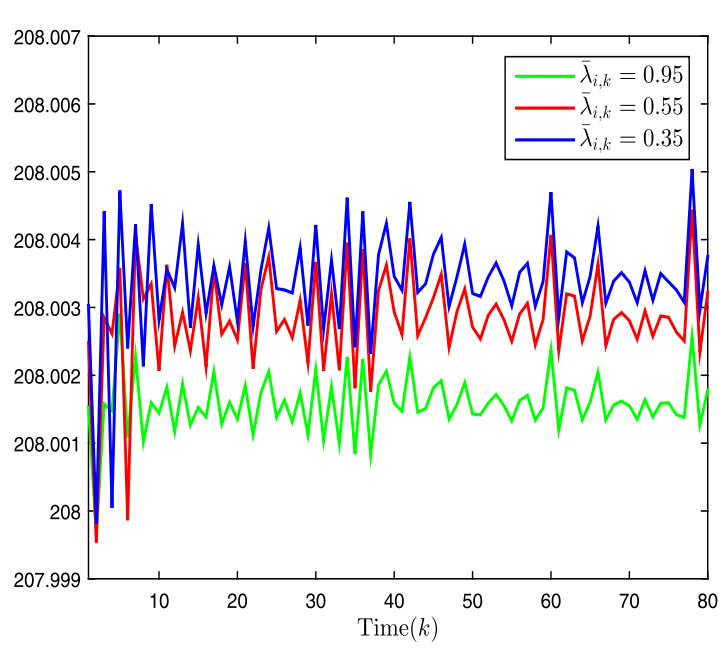

Figure 7 Upper bound with different $\bar{\lambda}_{i, k}$

Example 2 The corresponding parameters are given as follows:

$$
\begin{aligned}
& A_{k}=\left[\begin{array}{ccc}
0.15+0.45 \sin (0.1 k) & -1.7-0.2 \cos (0.98 k) & 0 \\
0.05 & -0.3-0.65 \cos (0.7 k) & 0 \\
0 & 0 & -0.7 \sin (k)
\end{array}\right], \\
& B_{k}=\left[\begin{array}{c}
0.3 \\
0.35 \\
0.4
\end{array}\right], \quad f\left(x_{k-d}\right)=\left[\begin{array}{c}
0.12-2.15 \cos \left(x_{2, k-d}\right) \\
0.24 x_{1, k-d}-1.15 \sin \left(x_{2, k-d}\right) \\
0.12-2.15 \cos \left(x_{3, k-d}\right)
\end{array}\right] .
\end{aligned}
$$

The initial values are set as $\bar{x}_{0}=\left[\begin{array}{lll}0 & 0 & 0\end{array}\right]^{T}, P_{0}=\operatorname{diag}\{2,2,2\}, \bar{x}_{-1}=\left[\begin{array}{lll}0 & 0 & 0\end{array}\right]^{T}, P_{-1}=$ $\operatorname{diag}\{1.5,2,2.5\}, \hat{x}_{1,0 \mid 0}=\hat{x}_{1,-1 \mid-1}=\left[\begin{array}{lll}-2.8 & 2.5 & 2\end{array}\right]^{T}, \hat{x}_{2,0 \mid 0}=\hat{x}_{2,-1 \mid-1}=\left[\begin{array}{lll}-2.3 & 2.2 & 2.1\end{array}\right]^{T}, \hat{x}_{3,0 \mid 0}=$ $\hat{x}_{3,-1 \mid-1}=\left[\begin{array}{lll}-2.3 & 2.3 & 2.1\end{array}\right]^{T}, \hat{x}_{4,0 \mid 0}=\hat{x}_{4,-1 \mid-1}=\left[\begin{array}{lll}-2.2 & 2.5 & 2\end{array}\right]^{T}, \Sigma_{1,0 \mid 0}=\operatorname{diag}\{15,15,15\}, \Sigma_{2,0 \mid 0}=$ $\operatorname{diag}\{25,25,25\}, \Sigma_{3,0 \mid 0}=\operatorname{diag}\{35,35,35\}$, and $\Sigma_{4,0 \mid 0}=\operatorname{diag}\{45,45,45\} ; \varsigma_{3, \max }=4$. The other parameters are same as in Example 1.

Again, the corresponding simulations can be obtained by implementing the proposed DEKF algorithm. Accordingly, Figs. 8-10 plot the trajectories of the real state and their estimates, respectively. It is clear that the newly presented estimation algorithm performs well. Moreover, in order to illustrate the differences for system with different dimensions, it is observed that the CPU time in Example 1 is $182 \mathrm{~s}$ and the CPU time in Example 2 is $193 \mathrm{~s}$. Then, it is not difficult to find that the processing time will increase when the system dimension increases. The major reason is that the complex coupling relationships of nodes are involved and additional computations should be made.

\section{Conclusions}

In this paper, the problems of DEKF scheme and its algorithm performance evaluation have been discussed for SSNSs subject to state delay and ROCAs with UOPs. In particular, the attacks including FDIAs and DoSAs have been considered and the case of UOPs has been depicted by the combination of nominal means and error upper bounds. Subsequently, the major effort has been made to design a filter in a distributed manner and 


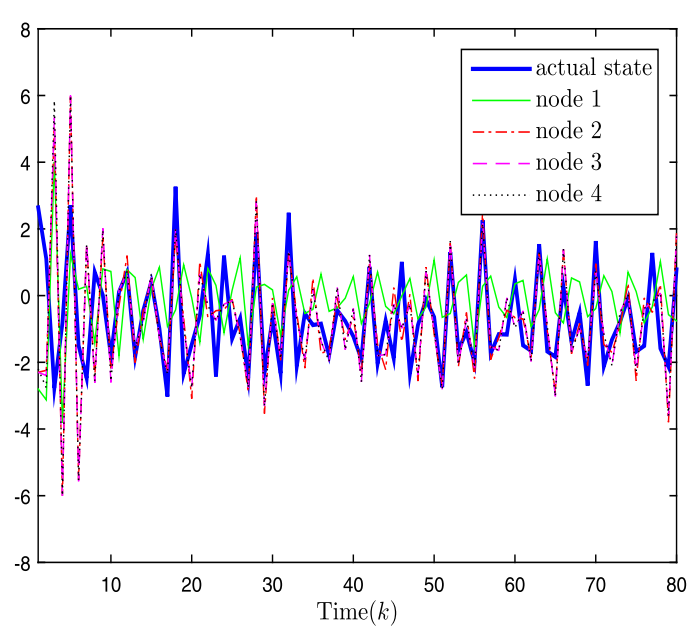

Figure 8 The actual state $x_{1, k}$ and the corresponding estimates

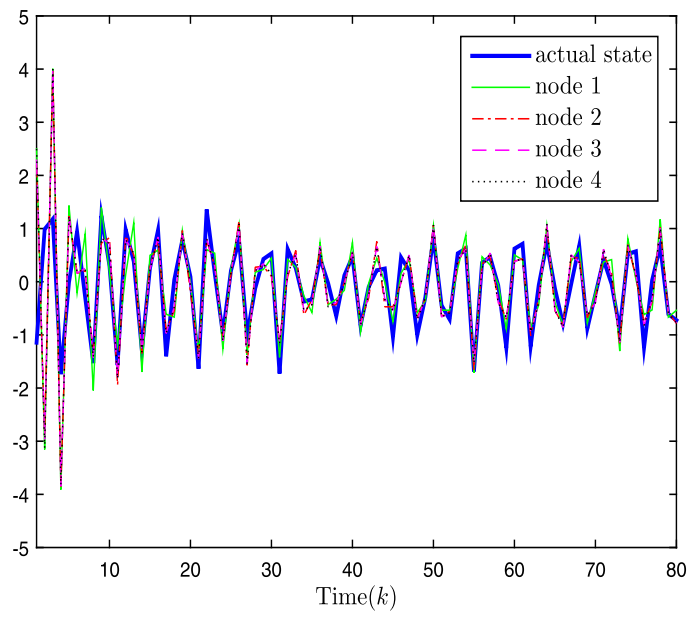

Figure 9 The actual state $x_{2, k}$ and the corresponding estimates

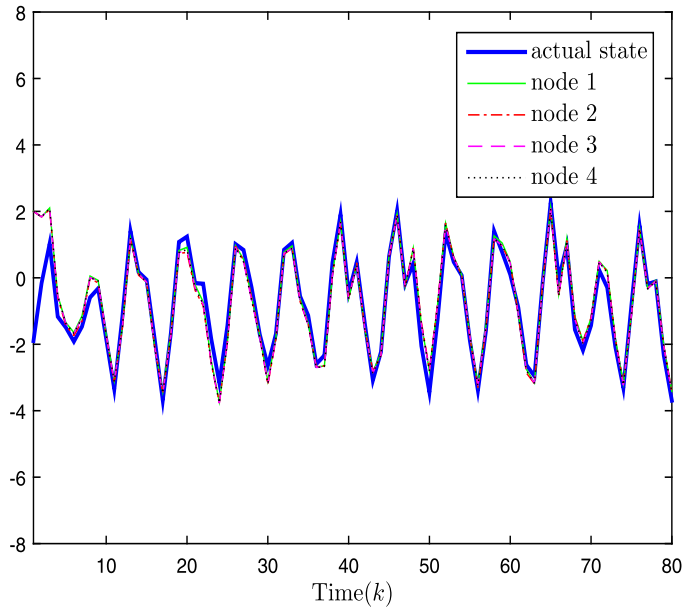

Figure 10 The actual state $x_{3, k}$ and the corresponding estimates 
look for an optimized upper bound matrix regarding the filtering error covariance. Furthermore, a suitable filter parameter has been designed at each time step to minimize the obtained upper bound matrix in the sense of matrix trace. Besides, the boundedness analysis has been certified for the DEKF algorithm by exploiting the elaborate mathematical methods. Finally, we have utilized two illustrative examples and comparative results to demonstrate the usefulness and advantages of the new DEKF approach.

\section{Acknowledgements}

The authors would like to appreciate the Associated Editor and anonymous reviewers for their helpful comments/suggestions on improving this paper.

\section{Funding}

This work was supported in part by the Outstanding Youth Science Foundation of Heilongjiang Province of China under grant JC2018001, the National Natural Science Foundation of China under Grant 61673141, the Fundamental Research Foundation for Universities of Heilongjiang Province of China under Grant 2019-KYYWF-0215, the European Regional Development Fund and Sêr Cymru Fellowship under Grant 80761-USW-059, the Fok Ying Tung Education Foundation of China under Grant 172034, and the Alexander von Humboldt Foundation of Germany.

\section{Availability of data and materials}

Not applicable.

\section{Competing interests}

All authors declared that they have no competing interests.

\section{Consent for publication}

All authors read and approved the final version of the paper.

\section{Authors' contributions}

The authors contributed equally to this paper. All authors read and approved the final manuscript.

\section{Author details}

${ }^{1}$ Department of Mathematics, Harbin University of Science and Technology, Harbin 150080, China. ${ }^{2}$ Heilongjiang Provincial Key Laboratory of Optimization Control and Intelligent Analysis for Complex Systems, Harbin University of Science and Technology, Harbin 150080, China. ${ }^{3}$ School of Engineering, University of South Wales, Pontypridd CF37 1DL, UK.

\section{Publisher's Note}

Springer Nature remains neutral with regard to jurisdictional claims in published maps and institutional affiliations.

Received: 17 March 2020 Accepted: 13 August 2020 Published online: 25 August 2020

\section{References}

1. Yick, J., Mukherjee, B., Ghosal, D.: Wireless sensor network survey. Comput. Netw. 52(12), 2292-2330 (2008)

2. Hu, J., Wang, Z., Liu, G.-P., Zhang, H.: Variance-constrained recursive state estimation for time-varying complex networks with quantized measurements and uncertain inner coupling. IEEE Trans. Neural Netw. Learn. Syst. 31(6), 1955-1967 (2020)

3. Hossein, S.M., De, D., Mohapatra, P.K.D., Mondal, S.P., Ahmadian, A., Ghaemi, F., Senu, N.: DNA sequences compression by $G P^{2} R$ and selective encryption using modified RSA technique. IEEE Access 8, 76880-76895 (2020)

4. Das, J.C., De, D., Mondal, S.P., Ahmadian, A., Ghaemi, F., Senu, N.: QCA based error detection circuit for nano communication network. IEEE Access 7, 67355-67366 (2019)

5. Wu, Y., Xia, W., Cao, M., Sun, X.-M.: Reach control problem for affine multi-agent systems on simplices. Automatica 107, 264-271 (2019)

6. Wu, Y., Sun, X.-M., Zhao, X., Shen, T.: Optimal control of Boolean control networks with average cost: a policy iteration approach. Automatica 100, 378-387 (2019)

7. Salahshoor, K., Mosallaei, M., Bayat, M.: Centralized and decentralized process and sensor fault monitoring using data fusion based on adaptive extended Kalman filter algorithm. Measurement 41(10), 1059-1076 (2008)

8. Rahmani, M., Abolhasani, M.: State estimation for stochastic time-varying multisensor systems with multiplicative noises: centralized and decentralized data fusion. Asian J. Control 21(4), 1547-1555 (2019)

9. Dong, H., Wang, Z., Gao, H.: Distributed $H_{\infty}$ filtering for a class of Markovian jump nonlinear time-delay systems over lossy sensor networks. IEEE Trans. Ind. Electron. 60(10), 4665-4672 (2012)

10. Li, W., Jia, Y., Du, J.: Distributed filtering for discrete-time linear systems with fading measurements and time-correlated noise. Digit. Signal Process. 60, 211-219 (2017)

11. Wen, C., Wang, Z., Geng, T., Alsaadi, F.E.: Event-based distributed recursive filtering for state-saturated systems with redundant channels. Inf. Fusion 39, 96-107 (2018)

12. Ma, L., Wang, Z., Liu, Y., Alsaadi, F.E.: Distributed filtering for nonlinear time-delay systems over sensor networks subject to multiplicative link noises and switching topology. Int. J. Robust Nonlinear Control 29(10), 2941-2959 (2019) 
13. Yuan, Y., Zhang, P., Wang, Z., Guo, L.: On resilient strategy design of multi-tasking optimal control for state-saturated systems with nonlinear disturbances: the time-varying case. Automatica 107, 138-145 (2019)

14. Selvaraj, P., Sakthivel, R., Ahn, C.K.: Observer-based synchronization of complex dynamical networks under actuator saturation and probabilistic faults. IEEE Trans. Syst. Man Cybern. Syst. 49(7), 1516-1526 (2018)

15. Ding, D., Wang, Z., Hu, J., Shu, H.: Dissipative control for state-saturated discrete time-varying systems with randomly occurring nonlinearities and missing measurements. Int. J. Control 86(4), 674-688 (2013)

16. Yuan, Y., Wang, Z., Zhang, P., Liu, H.: Near-optimal resilient control strategy design for state-saturated networked systems under stochastic communication protocol. IEEE Trans. Cybern. 49(8), 3155-3167 (2018)

17. Ding, D., Wang, Z., Shen, B., Shu, H.: State-saturated $H_{\infty}$ filtering with randomly occurring nonlinearities and packet dropouts: the finite-horizon case. Int. J. Robust Nonlinear Control 23(16), 1803-1821 (2013)

18. Li, X., Dong, H., Wang, Z., Han, F.: Set-membership filtering for state-saturated systems with mixed time-delays under weighted try-once-discard protocol. IEEE Trans. Circuits Syst. II, Express Briefs 66(2), 312-316 (2018)

19. Wen, C., Wang, Z., Liu, Q., Alsaadi, F.E.: Recursive distributed filtering for a class of state-saturated systems with fading measurements and quantization effects. IEEE Trans. Syst. Man Cybern. Syst. 48(6), 930-941 (2018)

20. Hu, J., Zhang, P., Kao, Y., Liu, H., Chen, D.: Sliding mode control for Markovian jump repeated scalar nonlinear systems with packet dropouts: the uncertain occurrence probabilities case. Appl. Math. Comput. 362, Article ID 124574 (2019). https://doi.org/10.1016/j.amc.2019.124574

21. Tong, M., Lin, W., Huo, X., Jin, Z., Miao, C.: A model-free fuzzy adaptive trajectory tracking control algorithm based on dynamic surface control. Int. J. Adv. Robot. Syst. 17(1), Article ID 1729881419894417 (2020). https://doi.org/10.1177/1729881419894417

22. Hu, J., Wang, Z., Gao, H.: Joint state and fault estimation for time-varying nonlinear systems with randomly occurring faults and sensor saturations. Automatica 97, 150-160 (2018)

23. Sakthivel, R., Vadivel, P., Mathiyalagan, K., Arunkumar, A., Sivachitra, M.: Design of state estimator for bidirectional associative memory neural networks with leakage delays. Inf. Sci. 296, 263-274 (2015)

24. Hu, J., Wang, Z., Liu, G.-P., Jia, C., Williams, J.: Event-triggered recursive state estimation for dynamical networks under randomly switching topologies and multiple missing measurements. Automatica 115, Article ID 108908 (2020). https://doi.org/10.1016/j.automatica.2020.108908

25. Mao, J., Ding, D., Song, Y., Liu, Y., Alsaadi, F.E.: Event-based recursive filtering for time-delayed stochastic nonlinear systems with missing measurements. Signal Process. 134, 158-165 (2017)

26. Chen, W., Ding, D., Dong, H., Wei, G.: Distributed resilient filtering for power systems subject to denial-of-service attacks. IEEE Trans. Syst. Man Cybern. Syst. 49(8), 1688-1697 (2019)

27. Lu, A., Yang, G.: False data injection attacks against state estimation in the presence of sensor failures. Inf. Sci. 508 92-104 (2020)

28. Shen, B., Wang, Z., Wang, D., Li, Q.: State-saturated recursive filter design for stochastic time-varying nonlinear complex networks under deception attacks. IEEE Trans. Neural Netw. Learn. Syst. (2019). https://doi.org/10.1109/TNNLS.2019.2946290

29. Liu, S., Wei, G., Song, Y., Liu, Y.: Extended Kalman filtering for stochastic nonlinear systems with randomly occurring cyber attacks. Neurocomputing 207, 708-716 (2016)

30. $\mathrm{Hu}$, J., Wang, Z., Alsaadi, F.E., Hayat, T.: Event-based filtering for time-varying nonlinear systems subject to multiple missing measurements with uncertain missing probabilities. Inf. Fusion 38, 74-83 (2017)

31. Gao, H., Lam, J., Wang, C., Wang, Y.: Delay-dependent output-feedback stabilisation of discrete-time systems with time-varying state delay. IEE Proc., Control Theory Appl. 151(6), 691-698 (2004)

32. Luo, M., Zhong, S., Cheng, J.: Finite-time event-triggered control and fault detection for singular Markovian jump mixed delay systems under asynchronous switching. Adv. Differ. Equ. 2018, Article ID 80 (2018). https://doi.org/10.1186/s13662-018-1533-y

33. Wang, B., Zou, F.C., Cheng, J., Zhong, S.M.: Fault detection filter design for continuous-time nonlinear Markovian jump systems with mode-dependent delay and time-varying transition probabilities. Adv. Differ. Equ. 2017, Article ID 262 (2017). https://doi.org/10.1186/s13662-017-1313-0

34. Hu, J., Zhang, H., Yu, X., Liu, H., Chen, D.: Design of sliding-mode-based control for nonlinear systems with mixed-delays and packet losses under uncertain missing probability. IEEE Trans. Syst. Man Cybern. Syst. (2019). https://doi.org/10.1109/TSMC.2019.2919513

35. Zou, L., Wang, Z., Gao, H., Liu, X.: State estimation for discrete-time dynamical networks with time-varying delays and stochastic disturbances under the round-robin protocol. IEEE Trans. Neural Netw. Learn. Syst. 28(5), 1139-1151 (2017)

36. Chen, D., Chen, W., Hu, J., Liu, H.: Variance-constrained filtering for discrete-time genetic regulatory networks with state delay and random measurement delay. Int. J. Syst. Sci. 50(2), 231-243 (2019)

37. Dadkhah, E., Shiri, B., Ghaffarzadeh, H., Baleanu, D.: Visco-elastic dampers in structural buildings and numerical solution with spline collocation methods. J. Appl. Math. Comput. 63, 29-57 (2020)

38. Khiabani, E.D., Ghaffarzadeh, H., Shiri, B., Katebi, J.: Spline collocation methods for seismic analysis of multiple degree of freedom systems with visco-elastic dampers using fractional models. J. Vib. Control (2020). https://doi.org/10.1177/1077546319898570

39. Shiri, B., Baleanu, D.: System of fractional differential algebraic equations with applications. Chaos Solitons Fractals $120,203-212(2020)$

40. Shiri, B., Baleanu, D.: Numerical solution of some fractional dynamical systems in medicine involving non-singular kernel with vector order. Results Nonlinear Anal. 2(4), 160-168 (2019)

41. Ma, C.-Y., Shiri, B., Wu, G.-C., Baleanu, D.: New signal smoothing equations with short memory and variable order Optik (2020). https://doi.org/10.1016/j.ijleo.2020.164507

42. Baleanu, D., Shiri, B.: Collocation methods for fractional differential equations involving non-singular kernel. Chaos Solitons Fractals 116, 136-145 (2018) 